\title{
SAMPLING ALMOST PERIODIC AND RELATED FUNCTIONS
}

\author{
STEFANO FERRI, JORGE GALINDO, AND CAMILO GÓMEZ
}

\begin{abstract}
We consider certain finite sets of circle-valued functions defined on intervals of real numbers and estimate how large the intervals must be for the values of these functions to be uniformly distributed in an approximate way. This is used to establish some general conditions under which a random construction introduced by Katznelson for the integers yields sets that are dense in the Bohr group. We obtain in this way very sparse sets of real numbers (and of integers) on which two different almost periodic functions cannot agree, what makes them amenable to be used in sampling theorems for these functions. These sets can be made as sparse as to have zero asymptotic density or as to be t-sets, i.e., to be sets that intersect any of their translates in a bounded set. Many of these results are proved not only for almost periodic functions but also for classes of functions generated by more general complex exponential functions, including chirps.
\end{abstract}

\section{INTRODUCTION}

Many sampling processes depend on choosing a sampling set where the functions to be sampled are uniquely determined. In the case of almost periodic functions on $\mathbb{R}$, sets with that property admit a neat topological description: they are precisely those subsets of $\mathbb{R}$ that are dense in the Bohr topology. A clear separation between consecutive samples is another natural prerequisite and, by this reason, sampling sets are usually required to be uniformly discrete. Although, Bohr-density and uniform discreteness might strike as conflicting requirements, they are not totally incompatible: Bohr-dense sets can be not only uniformly discrete but even quite sparse, as we next describe.

Collet [3] (see also [2]) proved that a random selection of points in regularly spaced time-windows almost surely produces a set that is uniformly distributed in the Bohr compactification, hence dense in the Bohr topology (see the section below for unexplained terms), of asymptotic density as small as desired.

Concentrating on a different sort of problems, Katznelson [9] devised a method that produces subsets of the group of integers $\mathbb{Z}$ with asymptotic density 0 which are dense in the Bohr topology. These constructions were further developed in [7, 11].

In this paper we lay out general conditions for Katznelson's method to work both in $\mathbb{R}$ and $\mathbb{Z}$ for almost periodic functions and, more generally, for function spaces generated by

2010 Mathematics Subject Classification. Primary 43A60; Secondary 11K70,42A75; 94A20.

Key words and phrases. Almost periodic function, Bohr topology, matching set, sampling set, chirp, discrepancy, uniform distribution, sampling, t-set, Bohr-dense.

The support of research project P1·1B2014-35 (Universitat Jaume I) is gratefully acknowledged.

Research of the first named author supported by the Faculty of Sciences of Universidad de los Andes via the Proyecto Semilla: "Representabilidad de grupos topológicos y de Álgebras de Banach y aplicaciones". Research of the second named author supported by Ministerio de Economía y Competitividad (Spain) through project MTM2016-77143-P (AEI/FEDER, UE).

The support is gratefully acknowledged. 
complex exponentials $e^{2 \pi i p(t)}$ with $p(t)$ running over sets of polynomials of bounded degree with coefficients in $\mathbb{R}$. These conditions are established in Theorems 4.2 and 4.4.

The distribution of $e^{2 \pi i p_{1}(t)}, \ldots, e^{2 \pi i p_{k}(t)}$ in the unit circle when $t$ takes values in the sampling interval is a key concept in our results, hence the relation with uniform distribution that appears in Section 3.

The dense sets we obtain, as those in $[2,3,9]$, come from a Borel-Cantelli argument that might require extremely large intervals to be reliable. Our approach gives hints on the minimum size a sampling interval must be in order to use our methods for approximate sampling. These estimates become quite concrete when the sampled function is extracted from a finite set of rational characters, see Corollary 3.9.

Once our general construction is laid, dense subsets of the Bohr group with specific properties are easy to obtain. In order to illustrate this, we show the existence of $t-$ sets that are dense in the Bohr compactification. By a t-set we refer here to a special sort of thin sets, introduced by Rudin [14], which are sets of interpolation for the weakly almost periodic functions, see Section 6 for more on this.

1.1. Notation and terminology. We collect in this section our notation for the standard concepts that will appear throughout the paper.

1.1.1. Topological groups. Even if most of our results are proved for $G=\mathbb{R}$ or $G=\mathbb{Z}$, we shall be keeping track of those that hold true in a more general setting. Accordingly, the results of Section 2 are proved for locally compact Abelian (LCA) groups or general topological Abelian groups. All groups will be assumed to be Abelian and additive notation will be used (and that includes using 0 as neutral element), with the notable exception of the circle group $\mathbb{T}$ that stands for the multiplicative group of complex numbers of modulus one. It will be usually represented as $\mathbb{T}=\left\{e^{2 \pi i s}: s \in[0,1)\right\}$. Multiplicative notation will also be used for groups of $\mathbb{T}$-valued functions.

1.1.2. Haar measure. The Haar measure of a LCA group $G$ will be denoted by $\lambda_{G}$. Haar measures will be normalized so that $\lambda_{G}$ corresponds to counting measure for $G=\mathbb{Z}$, to Lebesgue measure for $G=\mathbb{R}$, and to the invariant measure with $\lambda_{K}(K)=1$ for a compact group $K$. If $I \subseteq \mathbb{R}$ is an interval, $\lambda_{\mathbb{R}}(I)$ will therefore denote the length of $I$.

1.1.3. Characters and almost periodic functions. If $G$ is a topological group, $C(G, \mathbb{T})$ will stand for the multiplicative group of continuous $\mathbb{T}$-valued functions, and $\widehat{G}$ for its subgroup made of continuous homomorphisms. We shall refer to $\widehat{G}$ as the character group of $G$. Characters of $\mathbb{R}$ will be denoted by $\chi_{\tau}, \tau \in \mathbb{R}$ where $\chi_{\tau}(s)=e^{2 \pi i \tau s}$, for every $s \in \mathbb{R}$. It is a standard fact that the map $\tau \longmapsto \chi_{\tau}$ establishes an isomorphism which maps $\mathbb{R}$ onto $\widehat{\mathbb{R}}$. The same mapping with $\tau \in \mathbb{Z}$ establishes an isomorphism which maps $\mathbb{Z}$ onto $\widehat{\mathbb{T}}$.

If $p(x)$ is a polynomial with real coefficients, $\psi_{p}$ will denote the function $\psi_{p}(t)=e^{2 \pi i p(t)}$. If $p(t)=\tau t$, then $\psi_{p}=\chi_{\tau}$. The symbol $\mathfrak{C}_{n}$ will stand for the set $\left\{e^{2 \pi i p(t)}: p(x) \in \mathbb{R}_{n}[x]\right\}$, where $\mathbb{R}_{n}[x]$ denotes the set of polynomials in $\mathbb{R}[x]$ of degree at most $n$.

Characters are the main building blocks of almost periodic functions. Following H. Bohr's original definition, we can define almost periodic functions as uniform limits of linear combinations of characters (also known as trigonometric polynomials). Even if this was Bohr's original definition, other definitions of almost periodicity which do not make reference to trigonometric polynomials have been adopted later on, see $[8$, Theorem VI.5.21] for their equivalence. 
The topology that almost periodic functions induce on a group is known as the Bohr topology. This is the topology the group inherites from its embedding in $\prod_{\chi \in \widehat{G}} \mathbb{T}_{\chi}$, with $\mathbb{T}_{\chi}=\mathbb{T}$ for every $\chi$, given by $g \longmapsto(\chi(g))_{\chi}$ for every $g \in G$. The closure $G^{\mathcal{A P}}$ of $G$ in $\prod_{\chi \in \widehat{G}} \mathbb{T}_{\chi}$ is known as the Bohr compactification of $G$ and can also be identified with the spectrum of the Banach algebra $\mathcal{A P}(G)$ of almost periodic functions. A function $f \in C_{b}(G)$ is almost periodic precisely when it can be continuously extended to a function $f^{b} \in C\left(G^{\mathcal{A P}}\right)$. Finding discrete subsets of $G$ which are dense in $G^{\mathcal{A P}}$ permits then to have a single possible extension $f^{b}$ (and, as a consequence, a single $f$ ) that fits with the values it takes on these points.

The type of functions suitable for our sampling methods are those which have summable Bohr-Fourier series. We next extend the definitions of almost periodic functions, and functions with summable Bohr-Fourier series to the broader context we deal with which includes spaces generated by other $\mathbb{T}$-valued functions.

Definition 1.1. Let $G$ be a topological group. For $\mathfrak{J} \subseteq C(G, \mathbb{T})$, let $\operatorname{span}(\mathfrak{J})$ denote the linear span of $\mathfrak{J}$ in the vector space $C(G, \mathbb{C})$. We define:

$$
\begin{aligned}
\mathcal{A} \mathcal{P}_{\mathfrak{J}}(G) & =\overline{\operatorname{span}(\mathfrak{J})}\|\cdot\|_{\infty}, \text { and } \\
A_{\mathfrak{J}}(G) & =\left\{\sum_{\phi \in \mathfrak{J}} \alpha_{\phi} \phi: \sum_{\phi \in \mathfrak{J}}\left|\alpha_{\phi}\right|<\infty\right\} .
\end{aligned}
$$

The natural isomorphism between the Banach algebra $\ell_{1}(\mathfrak{J})$ and $A_{\mathfrak{J}(G)}$ defines a norm on this latter space. We denote this norm by $\|\cdot\|_{A_{\mathfrak{J}}}$.

For $\mathfrak{J}=\widehat{G}, \mathcal{A P}_{\mathfrak{J}}(G)=\mathcal{A P}(G)$ and $A_{\mathfrak{J}}(G)$ can be identified with the Fourier algebra $A\left(G^{\mathcal{A P}}\right)$ (as defined, for instance, in [15, Section 1.2.3]) on the Bohr compactificaction $G^{\mathcal{A P}}$ of $G$.

Note that $A_{\mathfrak{J}}(G)$ may be strictly contained in $\mathcal{A P}_{\mathfrak{J}}(G)$, see [15, Theorem 4.6.8], the result is originally due to Segal [16].

1.1.4. Uniform distribution. If $K$ is a compact group, a sequence $S=\left\{s_{n}: n \in \mathbb{N}\right\} \subset K$ is said to be uniformly distributed in $K$ (see [10, Definition 4.1.1]) if

$$
\lim _{N \rightarrow \infty} \frac{1}{N} \sum_{n=1}^{N} f\left(s_{n}\right)=\int_{K} f d \lambda_{K},
$$

for every continuous complex-valued function $f$ on $K$. This obviously implies that $S$ is dense in $K$.

A sequence $T=\left\{e^{2 \pi i s_{n}}: n \in \mathbb{N}\right\} \subset \mathbb{T}$, is uniformly distributed in $\mathbb{T}$ if and only if the sequence $S=\left\{s_{n}: n \in \mathbb{N}\right\}$ is uniformly distributed modulo 1 , i. e., every interval $[a, b) \subset[0,1]$ contains approximately $(b-a) N$ terms of the fractional part of the first $N$ terms of $S$.

1.1.5. Duality notations. When it comes to duality, the circle group $\mathbb{T}$ has a central rôle. When a specific distance on $\mathbb{T}$ is required our choice is the angular distance:

$$
d_{a}\left(e^{2 \pi i t}, e^{2 \pi i s}\right)=|t-s|, \quad \text { for every } s, t \in[0,1) .
$$

We define the open ball of radius $\varepsilon$ and center 1 as:

$$
\mathcal{V}_{\varepsilon}=\left\{e^{2 \pi i t} \in \mathbb{T}:|t|<\varepsilon\right\} \subset \mathbb{T} .
$$

Definition 1.2. For $I \subseteq G, a \in G, \Delta \subseteq C(G, \mathbb{T})$ and $\varepsilon>0$, we define: 
(1) $N^{\triangleright}(I, \varepsilon)=\left\{\phi \in C(G, \mathbb{T}): \phi(I) \subset \mathcal{V}_{\varepsilon}\right\}$

(2) $N^{\triangleleft}(\Delta, \varepsilon, a)=\left\{t \in G: \phi(t) \in \phi(a) \cdot \mathcal{V}_{\varepsilon}\right.$, for all $\left.\phi \in \Delta\right\}$. When $a=0$, we write $N^{\triangleleft}(\Delta, \varepsilon)$ instead of $N^{\triangleleft}(\Delta, \varepsilon, 0)$.

Observe that, when $\Delta$ is made of homomorphisms, i.e., when $\Delta \subset \widehat{G}$, then $N^{\triangleleft}(\Delta, \varepsilon, a)=$ $a+N^{\triangleleft}(\Delta, \varepsilon)$ for every $a \in G$.

We now name the families of functions that spread in an approximately uniform way on a given interval.

Definition 1.3. For $I \subseteq G, n \in \mathbb{N}, a \in G$ and $\varepsilon>0$, we define:

$$
\begin{aligned}
\mathfrak{P}_{I, a, n, \varepsilon} & =\left\{F \subseteq C(G, \mathbb{T}):|F|=n \text { and } \lambda_{G}\left(N^{\triangleleft}(F, \varepsilon, a) \cap I\right) \geq \varepsilon^{n} \lambda_{G}(I)\right\}, \\
\mathfrak{P}_{I, n, \varepsilon} & =\left\{F \subseteq C(G, \mathbb{T}):|F|=n \text { and } \lambda_{G}\left(N^{\triangleleft}(F, \varepsilon, a) \cap I\right) \geq \varepsilon^{n} \lambda_{G}(I) \text { for all } a \in G\right\} .
\end{aligned}
$$

The elements of the sets $\mathfrak{P}_{I, a, n, \varepsilon}$ are a sort of individualization of the discrepancy functions. Recall that the discrepancy function of a sequence $\left\{x_{n}: n \in \mathbb{N}\right\} \subset \mathbb{R}^{k}$ is:

$$
D_{N}\left(x_{1}, \ldots, x_{N}\right)=\sup _{I \subset \mathbb{T}^{k}}\left|\frac{\left|j \in\{1, \ldots, N\}: e^{2 \pi i x_{j}} \in I\right|}{N}-\lambda_{\mathbb{T}^{k}}(I)\right| .
$$

If the discrepancy of $\left\{x_{1}, \ldots, x_{N}\right\}$ is low enough, then $\left\{e^{2 \pi i x_{1} t}, \ldots, e^{2 \pi i x_{N} t}\right\} \in \mathfrak{P}_{I, a, N, \varepsilon}$ for every $a$ and $I$. See Section 3 for more on this relation.

1.1.6. Random choices. Let $G$ be a locally compact group with Haar measure $\lambda_{G}$ and let $I \subset G$ be a subset of finite measure. In many of our proofs we consider a random choice $\Lambda$ of, say, $\ell$-many elements of $I$ and we estimate the probability that $\Lambda$ belongs to a measurable subset $\mathcal{A}$ of $I^{\ell}$ that is invariant under permuting coordinates. This random choice, and its corresponding probability, is always to be understood in the probability space induced by $\lambda_{G^{\ell}}$ on $I^{\ell}$. Hence, for a given $\mathcal{A} \subseteq I^{\ell}$, we have that $\mathbb{P}(\{\Lambda: \Lambda \in \mathcal{A}\})=$ $\frac{\lambda_{G^{\ell}}(\mathcal{A})}{\lambda_{G}(I)^{\ell}}$.

\section{Approximately Bohr-Dense subsets}

We start by defining the basic concepts we are after.

\subsection{Some definitions.}

Definition 2.1. Let $\Lambda \subseteq G$ be a subset of a topological group $G$ and let $\mathcal{A}$ be a vector subspace of $C(G, \mathbb{C})$. We say that $\Lambda$ is a set of uniqueness for $\mathcal{A}$ if whenever $f \in \mathcal{A}$ satisfies $\left.f\right|_{\Lambda}=0$, then $f=0$.

The following terminology already announces our approach.

We start by defining approximately dense subsets.

Definition 2.2. Let $G$ be a topological group. Given $F \subset C(G, \mathbb{T}), I \subseteq G$ and $\varepsilon>0$, we say that $\Lambda \subseteq G$ is an $(F, I, \varepsilon)$-matching set if:

For any $a \in I$, there is $x_{a} \in \Lambda$ such that $\phi\left(x_{a}\right) \in \phi(a) \cdot \mathcal{V}_{\varepsilon}$, for any $\phi \in F$.

The above definition can be restated like this:

$\Lambda \subseteq G$ is an $(F, I, \varepsilon)$-matching set if:

$$
\text { For any } a \in I, \quad \Lambda \cap N^{\triangleleft}(F, \varepsilon, a) \neq \varnothing .
$$

Sets of approximate uniqueness will be called $\varepsilon$-sampling sets: 
Definition 2.3. Let $G$ be a topological group. Fix $I \subseteq G$ and $\mathfrak{J} \subset C(G, \mathbb{T})$. We say that $\Lambda \subseteq I$ is an $(\mathfrak{J}, I, \varepsilon)$-sampling set if for any $f \in A_{\mathfrak{J}}(G)$ one has that:

$$
\left\|\left.f\right|_{I}\right\|_{\infty} \leq \varepsilon\|f\|_{A_{\mathfrak{J}}}+\left\|\left.f\right|_{\Lambda}\right\|_{\infty} .
$$

2.2. Matching sets are sampling sets. We now see that matching sets, even when slightly thickened, are sampling sets.

Proposition 2.4. Let $G$ be a topological group, $\Lambda \subseteq I \subseteq G$ and $\mathfrak{J} \subseteq C(G, \mathbb{T})$.

Suppose that there is $F \subseteq C(G, \mathbb{T})$ that satisfies:

(1) $\Lambda$ is an $(F, I, \varepsilon)$-matching set, and

(2) $\mathfrak{J} \subset F \cdot N^{\triangleright}(I, \varepsilon)$.

Then $\Lambda$ is a $(\mathfrak{J}, I,(6 \pi+2) \varepsilon)$-sampling set.

Proof. Let $f \in A_{\mathfrak{J}}(G)$ with $\left\|\left.f\right|_{\Lambda}\right\|_{\infty} \leq \delta$.

Take $\phi_{1}, \ldots, \phi_{n} \in \mathfrak{J}$ and $\alpha_{1}, \ldots, \alpha_{n} \in \mathbb{C}$ so that

$$
\begin{gathered}
\left\|f-\sum_{j=1}^{N} \alpha_{j} \phi_{j}\right\|_{\infty}<\varepsilon\|f\|_{A_{\mathfrak{J}}} \text {, and } \\
\sum_{j=1}^{N}\left|\alpha_{j}\right|<\|f\|_{A_{\mathfrak{J}}} .
\end{gathered}
$$

Let $z \in I$ be fixed. Since $\mathfrak{J} \subset F \cdot N^{\triangleright}(I, \varepsilon)$ we can choose $\psi_{j} \in F$ and $\kappa_{j} \in N^{\triangleright}(I, \varepsilon)$ with $\phi_{j}=\psi_{j} \cdot \kappa_{j}$.

Find, according to the definition of $(F, I, \varepsilon)$-matching set, $x_{z} \in \Lambda$ such that

$$
\psi\left(x_{z}\right) \in \psi(z) \cdot \mathcal{V}_{\varepsilon} \text { for every } \psi \in F .
$$

Then:

$$
\begin{aligned}
|f(z)| & \leq\left|f(z)-\sum_{j=1}^{n} \alpha_{j} \phi_{j}(z)\right| \\
& +\left|\sum_{j=1}^{n} \alpha_{j} \phi_{j}(z)-\sum_{j=1}^{n} \alpha_{j} \psi_{j}(z)\right| \\
& +\left|\sum_{j=1}^{n} \alpha_{j} \psi_{j}(z)-\sum_{j=1}^{n} \alpha_{j} \psi_{j}\left(x_{z}\right)\right| \\
& +\left|\sum_{j=1}^{n} \alpha_{j} \psi_{j}\left(x_{z}\right)-\sum_{j=1}^{n} \alpha_{j} \phi_{j}\left(x_{z}\right)\right| \\
& +\left|\sum_{j=1}^{n} \alpha_{j} \phi_{j}\left(x_{z}\right)-f\left(x_{z}\right)\right| \\
& +\left|f\left(x_{z}\right)\right| .
\end{aligned}
$$

Now, observing that $\kappa_{j} \in N^{\triangleright}(I, \varepsilon)$ implies that $\left|\kappa_{j}(z)-1\right|<2 \pi \varepsilon$ : 


$$
\begin{aligned}
\left|\sum_{j=1}^{n} \alpha_{j} \phi_{j}(z)-\sum_{j=1}^{n} \alpha_{j} \psi_{j}(z)\right| & \leq \sum_{j=1}^{n}\left|\alpha_{j}\right|\left|\phi_{j}(z)-\psi_{j}(z)\right| \\
& =\sum_{j=1}^{n}\left|\alpha_{j}\right|\left|\psi_{j}(z) \kappa_{j}(z)-\psi_{j}(z)\right| \\
& =\sum_{j=1}^{n}\left|\alpha_{j}\right|\left|\kappa_{j}(z)-1\right| \\
& \leq \sum_{j=1}^{n}\left|\alpha_{j}\right| 2 \pi \varepsilon<2 \pi \varepsilon \cdot\|f\|_{A_{\mathfrak{J}}} .
\end{aligned}
$$

Using (2.2), instead of $\kappa_{j} \in N^{\triangleright}(I, \varepsilon)$, yields

$$
\begin{aligned}
\left|\sum_{j=1}^{n} \alpha_{j} \psi_{j}(z)-\sum_{j=1}^{n} \alpha_{j} \psi_{j}\left(x_{z}\right)\right| & \leq \sum_{j=1}^{n}\left|\alpha_{j}\right|\left|\psi_{j}(z)-\psi_{j}\left(x_{z}\right)\right| \\
& \leq 2 \pi \varepsilon \cdot\|f\|_{A_{\mathfrak{J}}} .
\end{aligned}
$$

Applying (2.9), and its analog for $x_{z}$, to bound the differences (2.4) and (2.6), inequality (2.10) to bound the difference (2.5) and inequality (2.1) to bound the differences (2.3) and (2.7) and taking into account that $\left|f\left(x_{z}\right)\right|<\left\|\left.f\right|_{\Lambda}\right\|_{\infty}$ we conclude that

$$
|f(z)| \leq(6 \pi+2) \varepsilon\|f\|_{A_{\mathfrak{J}}}+\left\|\left.f\right|_{\Lambda}\right\|_{\infty} .
$$

As it may be expected, Proposition 2.4 yields density results when applied to all finite subsets of a concrete subspace of $C(G, \mathbb{T})$.

Corollary 2.5. Let $G$ be a topological group, $\mathfrak{J} \subseteq C(G, \mathbb{T})$ and $\Lambda \subseteq G$. Suppose that $\Lambda$ is an $(F, G, \varepsilon)$-matching set for every finite subset $F \subset \mathfrak{J}$ and every $\varepsilon>0$. Then $\Lambda$ is a set of uniqueness for $\mathcal{A P}_{\mathfrak{J}}(G)$.

Proof. Let $f \in \mathcal{A P}(G)$ with $\left.f\right|_{\Lambda}=0$ and let $0<\varepsilon<1$ be fixed.

Find a $\mathfrak{J}$-trigonometric polynomial

$$
P_{f}=\sum_{j=1}^{n} \alpha_{j} \psi_{j}
$$

with $\alpha_{j} \in \mathbb{C}$ and $\psi_{j} \in \mathfrak{J}, 1 \leq j \leq n$, such that:

$$
\left\|f-P_{f}\right\|_{\infty}<\varepsilon
$$

This implies that $\left\|\left.P_{f}\right|_{\Lambda}\right\|_{\infty} \leq \varepsilon$. Put $\tilde{\varepsilon}=\frac{\varepsilon}{\sum_{j=1}^{n}\left|\alpha_{j}\right|}$ and $F=\left\{\psi_{j}: 1 \leq j \leq n\right\}$. By Proposition 2.4 the set $\Lambda$ is an $(F, G,(6 \pi+2) \tilde{\varepsilon})$-sampling set. Since $P_{f} \in A_{F}(G)$ we have as a consequence that

$$
\left\|P_{f}\right\|_{\infty} \leq(6 \pi+2) \tilde{\varepsilon} \sum_{j=1}^{n}\left|\alpha_{j}\right|+\varepsilon \leq(6 \pi+3) \varepsilon .
$$


Now, since $\left\|f-P_{f}\right\|_{\infty} \leq \varepsilon$, we deduce that $\|f\|_{\infty}<(6 \pi+4) \varepsilon$. We conclude that $f=0$, for $\varepsilon$ was arbitrary.

In the case of almost periodic functions, every continuous function on $G^{\mathcal{A P}}$ coincides with an almost periodic function on $G$, we obtain therefore that $\Lambda$ is dense in $G^{\mathcal{A P}}$.

Corollary 2.6. Let $G$ be an Abelian topological group and $\Lambda \subseteq G$. Suppose that $\Lambda$ is an $(F, G, \varepsilon)$-matching set for every finite subset $F \subset \widehat{G}$ and every $\varepsilon>0$. Then $\Lambda$ is a set of uniqueness for $\mathcal{A P}(G)$. In particular $\Lambda$ is dense in $G^{\mathcal{A P}}$.

Proof. The only difference with Corollary 2.5 resides in the density statement. Gelfand Representation identifies $\mathcal{A P}(G)$ with $C\left(G^{\mathcal{A P}}, \mathbb{C}\right)$. If $\Lambda$ is not dense in $G^{\mathcal{A P}}$, by Urysohn's Lemma there will be a nonconstant function $f: G^{\mathcal{A P}} \rightarrow \mathbb{C}$ that vanishes on $\Lambda$ which is impossible since $f$ is determined by its values on $\Lambda$.

2.3. Estimates. We now estimate the probability of selecting a matching set at some point $a \in G$ in a random selection of sampling points.

We first name the events whose probability we are estimating.

Definition 2.7. Let $G$ be a locally compact Abelian group, $I \subseteq G$ and $a \in G$. For $\Delta \subset C(G, \mathbb{T}), n, \ell \in \mathbb{N}$, and $\varepsilon>0$, we define $\mathcal{A}_{a, \Delta, n, \ell, \varepsilon, I}$ to be the set of all $\Lambda \subseteq I$ which satisfy the following properties:

(1.) $|\Lambda|=\ell$, and

(2.) $\Lambda \cap\left(N^{\triangleleft}(F, \varepsilon, a)\right) \neq \varnothing$ for every $F \subseteq \Delta$ with $F \in \mathfrak{P}_{I, a, n, \varepsilon}$.

Remark 2.8. In what follows we regard the sets $\mathcal{A}_{a, \Delta, n, \ell, \varepsilon, I}$ as events in the probability space determined by the restriction of $\lambda_{G^{\ell}}$ to $I^{\ell}$, see the remarks in Subsection 1.1.6.

As we see next, the definition of the sets $\mathfrak{P}_{I, a, n, \varepsilon}$ and $\mathcal{A}_{a, \Delta, n, \ell, \varepsilon, I}$ is tailored to facilitate these estimations.

Lemma 2.9. Let $I \subseteq G$ be a subset of positive Haar measure and $\Delta \subseteq C(G, \mathbb{T})$ be a finite set with $|\Delta|=N$. Consider as well $a \in G, \varepsilon>0$ and $n \in \mathbb{N}$. Then

$$
\mathbb{P}\left(\mathcal{A}_{a, \Delta, n, \ell, \varepsilon, I}^{\mathbf{c}}\right) \leq\left(\begin{array}{c}
N \\
n
\end{array}\right)\left(1-\varepsilon^{n}\right)^{\ell} \leq\left(\frac{N e}{n}\right)^{n}\left(1-\varepsilon^{n}\right)^{\ell}
$$

Proof. The second inequality is a well-known estimate of binomial numbers.

For the first inequality, we observe that

$$
\mathcal{A}_{a, \Delta, n, \ell, \varepsilon, I}^{\mathbf{c}}=\left\{\Lambda \subset I^{\ell}: \Lambda \notin \mathcal{A}_{a, \Delta, n, \ell, \varepsilon, I}\right\}=\bigcup_{\substack{F \subseteq \Delta \\ F \in \mathfrak{P}_{I, a, n, \varepsilon}}}\left(\left(N^{\triangleleft}(F, \varepsilon, a) \cap I\right)^{\mathbf{c}}\right)^{\ell} .
$$

By the very definition of $\mathfrak{P}_{I, a, n, \varepsilon}$, we have that $\lambda_{G}\left(\left(N^{\triangleleft}(F, \varepsilon, a) \cap I\right)^{\mathbf{c}}\right) \leq \lambda_{G}(I)-\varepsilon^{n} \lambda_{G}(I)$, hence $\mathbb{P}\left(\left(\left(N^{\triangleleft}(F, \varepsilon, a) \cap I\right)^{\mathbf{c}}\right)^{\ell}\right) \leq\left(1-\varepsilon^{n}\right)^{\ell}$. Since there are $\left(\begin{array}{l}N \\ n\end{array}\right)$ different subsets of $\Delta$ of cardinality $n$, the inequality follows.

Definition 2.10. Let $G$ be a $L C A$ group and let $I^{*}=\left\{I_{k}: k \in \mathbb{N}\right\}$ and $\Delta^{*}=\left\{\Delta_{k}: k \in \mathbb{N}\right\}$ be two sequences of subsets of $G$ and $C(G, \mathbb{T})$, respectively. Let as well a sequence of positive integers, $\ell^{*}=\left\{\ell_{k}: k \in \mathbb{N}\right\}, a \in G, n \in \mathbb{N}$ and $\varepsilon>0$ be given. We define:

$\mathcal{B}_{a, \Delta^{*}, n, \ell^{*}, \varepsilon, I^{*}}=\left\{\left(\Lambda_{k}\right)_{k}\right.$ : There exists $N \geq 1$ such that $\Lambda_{k} \in \mathcal{A}_{a, \Delta_{k}, n, \ell_{k}, \varepsilon, I_{k}}$ for all $\left.k \geq N\right\}$. 
Remark 2.11. We shall next estimate the probability of the events $\mathcal{B}_{a, \Delta^{*}, n, \ell^{*}, \varepsilon, I^{*}}$. These events are considered as events in the probability space $\prod_{k} I_{k}^{\ell_{k}}$ where each factor is assumed to carry the probability measure induced by the restriction of Haar measure on $G^{\ell_{k}}$, see the remarks in Subsection 1.1.6.

Lemma 2.12. Consider the sequences:

(1) $I^{*}=\left\{I_{k}: k \in \mathbb{N}\right\}$, with $I_{k} \subseteq G$ of nonzero Haar measure.

(2) $\Delta^{*}=\left(\Delta_{k}\right)_{k}$, with $\Delta_{k} \subset C(G, \mathbb{T})$ finite.

(3) $\ell^{*}=\left(\ell_{k}\right)_{k}$, with $\ell_{k} \in \mathbb{N}$.

Set $a \in G, n \in \mathbb{N}$ and $\varepsilon>0$.

If, for each $k, \Lambda_{k} \subseteq I_{k}$ is randomly chosen with $\left|\Lambda_{k}\right|=\ell_{k}$ and there is $k_{0}$ such that

$$
\ell_{k}>n \log \left(\left|\Delta_{k}\right|\right) \epsilon^{-n}-\frac{1}{\log \left(1-\varepsilon^{n}\right)} \log k
$$

for every $k \geq k_{0}$, then

$$
\mathbb{P}\left(\mathcal{B}_{a, \Delta^{*}, n, \ell^{*}, \varepsilon, I^{*}}\right)=1 .
$$

Proof. In this proof we identify the set $\mathcal{A}_{a_{k_{0}}, \Delta_{k_{0}}, n, \ell_{k_{0}, \varepsilon, I_{k_{0}}}}, k_{0} \in \mathbb{N}$ with the subset $\prod_{k} \mathcal{A}_{k} \subseteq$ $\prod_{k} I_{k}^{\ell_{k}}$ with $\mathcal{A}_{k_{0}}=\mathcal{A}_{a_{k_{0}}, \Delta_{k_{0}}, n, \ell_{k_{0}}, \varepsilon, I_{k_{0}}}$ and $\mathcal{A}_{k}=I_{k}^{\ell_{k}}$ for $k \neq k_{0}$. Note that the probability of the event $\mathcal{A}_{a_{k_{0}}, \Delta_{k_{0}}, n, \ell_{k_{0}}, \varepsilon, I_{k_{0}}}$ in the probability space $\prod_{k} I_{k}^{\ell_{k}}$ and in $I_{k_{0}}^{\ell_{k_{0}}}$ coincide.

Under this assumption we have that

$$
\mathcal{B}_{a, \Delta^{*}, n, \ell^{*}, \varepsilon, I^{*}}=\bigcup_{N=1}^{\infty} \bigcap_{k=N}^{\infty} \mathcal{A}_{a_{k}, \Delta_{k}, n, \ell_{k}, \varepsilon, I_{k}} .
$$

We now see that the complement of the latter event has probability 0 .

Putting $\delta_{n}:=1-\varepsilon^{n}, L_{k}:=\left|\Delta_{k}\right|$ and bearing in mind that $\log \delta_{n}<-\varepsilon^{n}$, it follows from Lemma 2.9 that

$$
\begin{aligned}
\sum_{k} \mathbb{P}\left(\left\{\Lambda: \Lambda \notin \mathcal{A}_{a, \Delta_{k}, n, \ell_{k}, \varepsilon, I_{k}}\right\}\right) & \leq \sum_{k}\left(\frac{L_{k} e}{n}\right)^{n} \delta_{n}^{\ell_{k}} \\
& =\left(\frac{e}{n}\right)^{n} \sum_{k} \delta_{n}^{\frac{n \log L_{k}}{\log \delta_{n}}+\ell_{k}} \\
& \leq\left(\frac{e}{n}\right)^{n} \sum_{k} \delta_{n}^{-n \varepsilon^{-n} \log L_{k}+\ell_{k}} \\
& \leq \sum_{k} \delta_{n}^{c \log k}<\infty
\end{aligned}
$$

where $c>-\frac{1}{\log \left(\delta_{n}\right)}$. Since $\delta_{n}^{-\frac{1}{\log \delta_{n}}}=\frac{1}{e}$ and the series $\sum_{k} x^{\log k}$ converges when $|x|<\frac{1}{e}$, the Borel-Cantelli Lemma shows that

$$
\mathbb{P}\left(\bigcap_{N=1}^{\infty} \bigcup_{k=N}^{\infty} \mathcal{A}_{a_{k}, \Delta_{k}, n, \ell_{k}, \varepsilon, I_{k}}^{\mathbf{c}}\right)=0 .
$$

Since $\mathcal{B}_{a, \Delta^{*}, n, \ell^{*}, \varepsilon, I^{*}}$ is precisely the complementary of this latter set, the lemma follows. 


\section{Matching intervals AND ChaRACTERS}

In this section $G=\mathbb{R}$ or $G=\mathbb{Z}$. Recall that for $p(x) \in \mathbb{R}[x], \psi_{p}(t)=e^{2 \pi i p(t)}$ and for $\tau \in \mathbb{R}, \chi_{\tau}(x)=e^{2 \pi i \tau x}$.

Our first task will be to see, given $F$, how long an interval $I$ has to be for $F \in \mathfrak{P}_{I, a, n, \varepsilon}$. This will be obtained as a consequence of the discrepancy of the functions in $F$.

Definition 3.1 (Definition 2.74 of [4]). Let $\mathbf{x}:[0,+\infty) \rightarrow \mathbb{R}^{n}$ be a continuous function. The continuous discrepancy of $\mathbf{x}$ in $[0, T]$ is defined by

$$
D_{T}(\mathbf{x})=\sup _{\mathcal{V} \subseteq \mathbb{T}^{n}}\left|\frac{1}{T} \int_{0}^{T} \mathbf{1}_{\mathcal{V}}\left(e^{2 \pi i \mathbf{x}(t)}\right) d t-\lambda_{\mathbb{T}^{n}}(\mathcal{V})\right|,
$$

where $\mathbf{1}_{\mathcal{V}}$ denotes the characteristic function of the set $\mathcal{V}, e^{2 \pi i \mathbf{x}(t)}$ stands for the vector $\left(e^{2 \pi i x_{1}(t)}, \ldots, e^{2 \pi i x_{n}(t)}\right) \in \mathbb{T}^{n}$ and the supremum is taken over all rectangles in $\mathbb{T}^{n}$ with sides parallel to the axes.

Definition 3.2 (Definition 2.70 and Theorem 2.75 of [4] ). A continuous function

$$
\mathbf{x}:[0,+\infty) \rightarrow \mathbb{R}^{n}
$$

is said to be continuously well distributed modulo 1 if $\lim _{T \rightarrow \infty} D_{T}(\mathbf{x}(t+\tau))=0$ uniformly on $\tau$.

We propose the following definition to make our notation lighter.

Definition 3.3. We say that the polynomials $p_{1}, \ldots, p_{n} \in \mathbb{R}[x]$ are strongly linearly independent over $\mathbb{Q}$ if for each nontrivial lattice point $\left(h_{1}, \ldots, h_{n}\right) \in \mathbb{Z}^{n}$ the polynomial $\sum_{j=1}^{n} h_{j} p_{j}$ is nonconstant.

The family $\left\{\psi_{p_{1}}, \ldots, \psi_{p_{n}}\right\}$ will be said to be strongly linearly independent over $\mathbb{Q}$ when $p_{1}, \ldots, p_{n}$ are strongly linearly independent over $\mathbb{Q}$.

Remark 3.4. Let $H$ be a Hamel basis of $\mathbb{R}$ over $\mathbb{Q}$ and let $C_{H}$ denote the constant polynomials with values in $H$. A set of polynomials $p_{1}, \ldots, p_{n} \in \mathbb{R}[x]$ is strongly linearly independent over $\mathbb{Q}$ if and only if the set $\left\{p_{1}, \ldots, p_{n}\right\} \cup C_{H}$ is linearly independent over $\mathbb{Q}$.

Theorem 3.5 (Corollary of Theorems 2.73 and 2.79 of [4]). If $p_{1}(t), \ldots, p_{n}(t)$ are strongly linearly independent polynomials over $\mathbb{Q}$ and $\mathbf{x}:[0,+\infty) \rightarrow \mathbb{R}^{n}$ is defined by $\mathbf{x}(t)=$ $\left(p_{1}(t), \ldots, p_{n}(t)\right)$, then $\mathbf{x}$ is continuously well distributed modulo 1.

Proof. By Weyl's criterion for continuous well-distribution [4, Theorem 2.73] we have to see that for every nonzero $\mathbf{h}=\left(h_{1}, \ldots, h_{n}\right) \in \mathbb{Z}^{n}$,

$$
\lim _{T \rightarrow \infty} \frac{1}{T} \int_{0}^{T} e^{2 \pi i \mathbf{h} \cdot x(t+\tau)} d t=0
$$

uniformly on $\tau \geq 0$.

If $\mathbf{h}=\left(h_{1}, \ldots, h_{n}\right) \in \mathbb{Z}^{n}$ is fixed then, as a consequence of the polynomials being strongly linearly independent over $\mathbb{Q}, q_{h}(t)=\sum_{j=1}^{n} h_{j} p_{j}(t)$ is a nonconstant polynomial. There are therefore $t_{0}$ and $C>0$ such that, for every $t \geq t_{0},\left|q_{h}(t)\right| \geq C$ and $q_{h}^{\prime \prime}(t)$ has constant sign. Theorem 2.79 of [4] then applies to show that $q_{h}(t)$ is continuously well distributed. Weyl's criterion applied to $q_{h}$ shows that (3.12) holds for this, and hence for all $\mathbf{h}$. 
Theorem 3.6. Let $F=\left\{\psi_{p_{1}}, \ldots, \psi_{p_{n}}\right\}$ where $\left\{p_{1}(x), \ldots, p_{n}(x)\right\}$ is a family of polynomials in $\mathbb{R}[x]$ that are strongly linearly independent over $\mathbb{Q}$ and let $\gamma>0$. Then there is $L(F, \gamma)$ such that, for every interval $I$ with $\lambda_{\mathbb{R}}(I) \geq L(F, \gamma)$ and every $a \in \mathbb{R}$ :

$$
\lambda_{\mathbb{R}}\left(N^{\triangleleft}(F, \delta, a) \cap I\right) \geq\left((2 \delta)^{n}-\gamma\right) \lambda_{\mathbb{R}}(I),
$$

for every $\delta>0$ such that $(2 \delta)^{n}-\gamma>0$. In particular, $F \in \mathfrak{P}_{I, n, \delta}$ whenever $\lambda_{\mathbb{R}}(I) \geq$ $L\left(F, \delta^{n}\left(2^{n}-1\right)\right)$.

Proof. Fix $a \in \mathbb{R}$ and $\gamma>0$. Define $\mathbf{x}_{F}:[0,+\infty) \rightarrow \mathbb{R}^{n}$ by $\mathbf{x}_{F}(t)=\left(p_{1}(t), \ldots, p_{n}(t)\right)$ and put, for each $\delta>0, \mathcal{V}_{\delta, a}=\left(\psi_{p_{1}}(a) \cdot \mathcal{V}_{\delta} \times \ldots \times \psi_{p_{n}}(a) \cdot \mathcal{V}_{\delta}\right) \subset \mathbb{T}^{n}$

By Theorem 3.5, the function $\mathbf{x}_{F}(t)$ is continuously well distributed. There is accordingly $L(F, \gamma)$ such that $T \geq L(F, \gamma)$ implies that $D_{T}(\mathbf{x}(t+\tau)) \leq \gamma$ for every $\tau$, i.e., $T \geq L(F, \gamma)$ implies that, for every $\tau$ :

$$
\frac{1}{T} \int_{0}^{T} \mathbf{1}_{\mathcal{V}_{a, \delta}}\left(e^{2 \pi i \mathbf{x}_{F}(t+\tau)}\right) d t \geq(2 \delta)^{n}-\gamma
$$

where $e^{2 \pi i \mathbf{x}_{F}(t+\tau)}$ is to be understood as in Definition 3.1.

Let now $I=\left[\tau_{0}, \tau_{0}+L\right]$ be an arbitrary interval of length $L \geq L(F, \gamma)$.

Taking into account the definition of $\mathcal{V}_{\delta, a}$, inequality (3.13), applied to $\tau=\tau_{0}$ and $T=L$, means that

$$
\begin{aligned}
L \cdot\left((2 \delta)^{n}-\gamma\right) & \leq \lambda_{\mathbb{R}}\left(\left\{t \in[0, L]: \psi_{p_{j}}\left(t+\tau_{0}\right) \in \psi_{p_{j}}(a) \cdot \mathcal{V}_{\delta}, \text { for } j=1, \ldots n\right\}\right) \\
& =\lambda_{\mathbb{R}}\left(\left\{t \in I: t \in N^{\triangleleft}(F, \delta, a)\right\}\right) .
\end{aligned}
$$

The same argument of Theorem 3.6 with well-distributed sequences instead of continuous well-distributed sequences can be used for $G=\mathbb{Z}$.

Corollary 3.7. Let $F=\left\{\psi_{p_{1}}, \ldots, \psi_{p_{n}}\right\}$ where $\left\{p_{1}(x), \ldots, p_{n}(x)\right\}$ is a family of polynomials in $\mathbb{R}[x]$ with coefficients in $[0,1)$ that are strongly linearly independent over $\mathbb{Q}$ and let $\gamma>0$. Then there is $L(F, \gamma)$ such that, for every interval $I \subseteq \mathbb{Z}$ with $|I| \geq L(F, \gamma)$ and every $a \in \mathbb{Z}$ :

$$
\left|N^{\triangleleft}(F, \delta, a) \cap I\right| \geq\left((2 \delta)^{n}-\gamma\right)|I|,
$$

for every $\delta>0$ such that $(2 \delta)^{n}-\gamma>0$. In particular, $F \in \mathfrak{P}_{I, n, \delta}$ whenever $\lambda_{\mathbb{R}}(I) \geq$ $L\left(F, \delta^{n}\left(2^{n}-1\right)\right)$.

3.1. Scholium: another proof of Theorem 3.6. When $F \subseteq \widehat{\mathbb{R}}$, we can provide a more constructive proof of Theorem 3.6 that can be used to estimate the value of $L(F, \gamma)$. This estimate is admittedly poor: if $H:=\frac{4}{e}\left(\frac{3}{2}\right)^{n}$, then $L(F, \gamma)=\frac{2}{e} S(H)\left(\frac{3}{2}\right)^{n}$, where $S(H)$ is as defined below.

Proof. (Second proof of Theorem 3.6 for $F=\left\{\chi_{\tau_{1}}, \ldots, \chi_{\tau_{n}}\right\}$ with $\tau_{1}, \ldots, \tau_{n} \in \mathbb{R}$ linearly independent over $\mathbb{Q}$.)

Let $I=\left[\tau_{0}, \tau_{0}+L\right]$ be an arbitrary interval. Define $\mathbf{x}_{F, I}:[0,+\infty) \rightarrow \mathbb{R}^{n}$ by

$$
\mathbf{x}_{F, I}(t)=\left(\left(\tau_{0}+t\right) \tau_{1}, \ldots,\left(\tau_{0}+t\right) \tau_{n}\right)
$$

and put $\mathcal{V}_{\delta, a}=\left(\psi_{p_{1}}(a) \cdot \mathcal{V}_{\delta} \times \cdots \times \psi_{p_{n}}(a) \cdot \mathcal{V}_{\delta}\right) \subset \mathbb{T}^{n}$ 
Apply the Erdôs-Turán-Koksma inequality to the above sequence. In the version of [4, Theorem 2.77] this implies that, for any positive integer $H$,

$$
D_{L}(\mathbf{x}) \leq\left(\frac{3}{2}\right)^{n}\left(\frac{2}{H+1}+\sum_{0<\|\mathbf{h}\|_{\infty} \leq H} \frac{1}{r(\mathbf{h})}\left|\frac{1}{L} \int_{0}^{L} e^{2 \pi i \mathbf{h} \cdot \mathbf{x}(t)} d t\right|\right),
$$

where $\mathbf{h} \cdot \mathbf{x}$ denotes scalar product, and $r(\mathbf{h})=\prod_{i=1}^{n} \max \left\{1,\left|h_{i}\right|\right\}$ for $\mathbf{h}=\left(h_{1}, \ldots, h_{n}\right) \in \mathbb{Z}^{n}$.

In the case at hand, $\mathbf{h} \cdot \mathbf{x}(t)=\left(\tau_{0}+t\right)\left(h_{1} \tau_{1}(t)+\cdots+h_{n} \tau_{n}(t)\right)$ and

$$
\left|\int_{0}^{L} e^{2 \pi i \mathbf{h} \cdot \mathbf{x}(t)} d t\right|=\left|\int_{0}^{L} e^{2 \pi i\left(\tau_{0}+t\right) \sum_{i=1}^{n} h_{i} \tau_{i}} d t\right| \leq \frac{1}{\pi\left|h_{1} \tau_{1}+\cdots+h_{n} \tau_{n}\right|} .
$$

Then, putting for each given $H$,

$$
S(H)=\sum_{0<\|\mathbf{h}\|_{\infty} \leq H} \frac{1}{r(\mathbf{h}) \cdot\left|h_{1} \tau_{1}+\cdots+h_{n} \tau_{n}\right|},
$$

inequality (3.14) turns into

$$
D_{L}(\mathbf{x}) \leq\left(\frac{3}{2}\right)^{n}\left(\frac{2}{H+1}+\frac{1}{L} S(H)\right) .
$$

If $H(n, \gamma)$ is then chosen so that $\left(\frac{3}{2}\right)^{n}\left(\frac{2}{H(n, \gamma)+1}\right) \leq \frac{\gamma}{2}$ and $L(F, \gamma)$ is chosen so that

$$
\left(\frac{3}{2}\right)^{n}\left(\frac{S(H(n, \gamma))}{L(F, \gamma)}\right) \leq \frac{\gamma}{2}
$$

we find that,

$$
D_{L}(\mathbf{x}) \leq \gamma \text { for every } L \geq L(F, \gamma) .
$$

Consider now an interval $I$ of length $\lambda_{\mathbb{R}}(I):=L \geq L(F, \varepsilon)$ and $\delta>0$. Then, by the definition of $D_{L}(\mathbf{x})$ :

$$
\int_{0}^{L} \mathbf{1}_{\mathcal{V}_{\delta}}\left(\mathbf{x}_{F, I}\right) d t \geq(2 \delta)^{n}-\gamma
$$

which is precisely the same inequality as (3.13), the proof proceeds then as in the case of Theorem 3.6.

3.2. Characters: some particular cases. In this subsection we shall be dealing with sets of characters instead of sets of more general continuous $\mathbb{T}$-valued functions. In this case $F \in \mathfrak{P}_{I, a, n, \varepsilon}$ if and only if $F \in \mathfrak{P}_{I, n, \varepsilon}$, so we shall only deal with the latter form.

For some special sets of characters $F$ we can actually find shorter intervals $I$ so that $F \in \mathfrak{P}_{I, n, \varepsilon}$.

Theorem 3.8. Let $0<\frac{q_{1}}{p_{1}}<\cdots<\frac{q_{n}}{p_{n}}=1$ be a finite sequence of rational numbers and let $0<\varepsilon<\frac{1}{2}$ be given. Put $N=p_{1} \cdots p_{n-1}$.

If $I \subset \mathbb{R}$ is an interval with $\lambda_{\mathbb{R}}(I) \geq N$, then $F:=\left\{\chi_{\frac{q_{j}}{p_{j}}}: 1 \leq j \leq n\right\} \in \mathfrak{P}_{I, n, \varepsilon}$.

Proof. We can assume that the fractions $\frac{q_{1}}{p_{1}}, \ldots, \frac{q_{n-1}}{p_{n-1}}$ are irreducible. If they are not we work with the simplified fractions and obtain a smaller $N$ that works.

We first assume that $\lambda_{\mathbb{R}}(I)=N$. 
For each $0 \leq j<p_{n-1}$, let

$$
L_{n-1, j}=\left\{z \in \mathbb{Z} \cap I: \frac{q_{n-1}}{p_{n-1}} \cdot z=\frac{j}{p_{n-1}}+\ell_{j} \text { for some } \ell_{j} \in \mathbb{Z}\right\} .
$$

Since (the class of) $q_{n-1}$ is a generator of the cyclic group $\mathbb{Z} / p_{n-1} \mathbb{Z}, L_{n-1, j}$ contains precisely $N / p_{n-1}$ integers with a distance of $p_{n-1}$ between consecutive ones.

If $z \in L_{n-1, j}$ and either $0 \leq j \leq\left\lfloor p_{n-1} \varepsilon\right\rfloor$ or $p_{n-1}-\left\lfloor p_{n-1} \varepsilon\right\rfloor \leq j<p_{n-1}$, an interval of length $\varepsilon$ (either $[z, z+\varepsilon]$ or $[z-\varepsilon, z]$ ) is contained in $I$ and sent into $\mathcal{V}_{\varepsilon}$ by both $\chi_{1}$ and $\chi_{\frac{q_{n-1}}{p_{n-1}}}$. If $z \in L_{n-1,0}$, then $\chi_{1}$ and $\chi_{\frac{q_{n-1}}{p_{n-1}}}$ map $z$ to 1 and, accordingly, $[z-\varepsilon, z+\varepsilon]$ into $\mathcal{V}_{\varepsilon}$

Define now, for every $m, 1 \leq m \leq n-1$ the set of positive integers:

$$
J_{n-m}=\left\{0,1, \ldots,\left\lfloor p_{n-m} \varepsilon\right\rfloor, p_{n-m}-\left\lfloor p_{n-m} \varepsilon\right\rfloor, \ldots, p_{n-m}-1\right\} .
$$

Note that $\left|J_{n-m}\right| \geq 2\left\lfloor p_{n-m} \varepsilon\right\rfloor+1$.

Claim: For each $(n-1)$-tuple $\left(j_{1}, \ldots, j_{n-1}\right) \in J_{1} \times \cdots \times J_{n-1}$ there is $z_{\left(j_{1}, \ldots, j_{n-1}\right)} \in \mathbb{Z}$ such that either $\left[z_{\left(j_{1}, \ldots, j_{n-1}\right)}, z_{\left(j_{1}, \ldots, j_{n-1}\right)}+\varepsilon\right] \subset N^{\triangleleft}(F, \varepsilon) \cap I$ or $\left[z_{\left(j_{1}, \ldots, j_{n-1}\right)}-\varepsilon, z_{\left(j_{1}, \ldots, j_{n-1}\right)}\right] \subset$ $N^{\triangleleft}(F, \varepsilon) \cap I$. In the case of $z_{(0, \ldots, 0)},\left[z_{(0, \ldots, 0)}-\varepsilon, z_{(0, \ldots, 0)}+\varepsilon\right] \subset N^{\triangleleft}(F, \varepsilon) \cap I$. The integers $z_{\left(j_{1}, \ldots, j_{n-1}\right)}$ are all different.

Fix, to start with, $j_{n-1} \in J_{n-1}$, arbitrarily.

For each $0 \leq j<p_{n-2}$, define

$$
L_{n-2, j}=\left\{z \in L_{n-1, j_{n-1}}: \frac{q_{n-2}}{p_{n-2}} \cdot z=\frac{j}{p_{n-2}}+\ell_{j} \text { for some } \ell_{j} \in \mathbb{Z}\right\} .
$$

As before, out of the $N / p_{n-1}$ elements of $L_{n-1, j_{n-1}}$, exactly $N /\left(p_{n-1} \cdot p_{n-2}\right)$ are in $L_{n-2, j}$. The distance between consecutive integers will be now $p_{n-1} \cdot p_{n-2}$.

If $0 \leq j \leq\left\lfloor p_{n-2} \varepsilon\right\rfloor$ or $p_{n-2}-\left\lfloor p_{n-2} \varepsilon\right\rfloor \leq j<p_{n-1}$ and $z \in L_{n-2, j}$, either the interval $[z, z+\varepsilon]$ or $[z-\varepsilon, z]$ is sent into $\mathcal{V}_{\varepsilon}$ by $\chi_{1}, \chi_{\frac{q_{n-1}}{p_{n-1}}}$ and $\chi_{\frac{q_{n-2}}{p_{n-2}}}$. If $j=0$, these characters map the whole of $[z-\varepsilon, z+\varepsilon]$ into $\mathcal{V}_{\varepsilon}$.

We next fix both $j_{n-1} \in J_{n-1}$ and $j_{n-2} \in J_{n-2}$, define $L_{n-3, j}$, and carry on the inductive process. After $(n-1)$ steps the integers $j_{n-1} \in J_{n-1}, j_{n-2} \in J_{n-2}, \ldots, j_{1} \in J_{1}$ will have been fixed. The set $L_{1, j}$ will contain precisely one integer, say $z_{\left(j_{1}, \ldots, j_{n-1}\right)} \in L_{1, j_{1}} \cap \cdots \cap$ $L_{n-1, j_{n-1}}$. Then:

$$
\text { either }\left[z_{\left(j_{1}, \ldots, j_{n-1}\right)}, z_{\left(j_{1}, \ldots, j_{n-1}\right)}+\varepsilon\right] \subseteq N^{\triangleleft}(F, \varepsilon) \cap I \text { or }\left[z_{\left(j_{1}, \ldots, j_{n-1}\right)}-\varepsilon, z_{\left(j_{1}, \ldots, j_{n-1}\right)}\right] \text {. }
$$

Since each $(n-1)$-tuple $\left(j_{1}, \ldots, j_{n-1}\right) \in J_{1} \times \cdots \times J_{n-1}$ produces a different $z_{\left(j_{1}, \ldots, j_{n-1}\right)}$ our claim is proved.

The intervals constructed in the previous claim have a total length of

$$
\varepsilon\left(\left(2\left\lfloor p_{1} \varepsilon\right\rfloor+1\right)\left(2\left\lfloor p_{2} \varepsilon\right\rfloor+1\right) \cdots\left(2\left\lfloor p_{n-1} \varepsilon\right\rfloor+1\right)+1\right) \geq 2 p_{1} \cdots p_{n-1} \varepsilon^{n},
$$

check the appendix to this section for a somewhat cumbersome, but otherwise routine, proof of the inequality.

We have thus shown that, when $\lambda_{\mathbb{R}}(I)=N$,

$$
\lambda_{\mathbb{R}}\left(N^{\triangleleft}(F, \varepsilon) \cap I\right) \geq 2 \lambda_{\mathbb{R}}(I) \varepsilon^{n},
$$

as desired.

Suppose now that $\lambda_{\mathbb{R}}(I)>N$. There are then $0<j \in \mathbb{N}$ and $0 \leq \delta<N$, such that $\lambda_{\mathbb{R}}(I)=j N+\delta$. Therefore, the interval $I$ can be split into $j$-many subintervals of length 
$N$ and another one of length $\delta N$. In each of the intervals of length $N$ we can argue as above and find a family of subintervals of $N^{\triangleleft}(F, \varepsilon) \cap I$ whose accumulated length is $2 N \varepsilon$.

We then obtain that

$$
\lambda_{\mathbb{R}}\left(N^{\triangleleft}(F, \varepsilon) \cap I\right) \geq 2 j N \varepsilon^{n} \geq 2 \varepsilon^{n}\left(1-\frac{1}{j+1}\right)(j N+\delta) \geq \varepsilon^{n} \lambda_{\mathbb{R}}(I) .
$$

Corollary 3.9. Let $0<\frac{q_{1}}{p_{1}}<\cdots<\frac{q_{n}}{p_{n}}$ be a finite sequence of rational numbers and let $0<\varepsilon<\frac{1}{2}$ be given. Put $N=p_{1} \cdots p_{n-1}$.

If $I \subset \mathbb{R}$ is an interval with $\lambda_{\mathbb{R}}(I) \geq N$, then $F:=\left\{\chi_{\frac{q_{j}}{p_{j}}}: 1 \leq j \leq n\right\} \in \mathfrak{P}_{I, n, \varepsilon}$.

Proof. Let $\tilde{F}=\left\{\chi_{\frac{p_{n} q_{1}}{q_{n} p_{1}}}, \ldots, \chi_{\frac{p_{n} q_{n-1}}{q_{n} p_{n-1}}}, \chi_{1}\right\}$.

Suppose $\lambda_{\mathbb{R}}(I) \geq p_{1} \cdots p_{n} \cdot q_{n}^{n-2}$. Then $\lambda_{\mathbb{R}}\left(\frac{q_{n}}{p_{n}} I\right) \geq q_{n}^{n-1} p_{1} \cdots p_{n-1}=\left(q_{n} p_{1}\right) \cdots\left(q_{n} p_{n-1}\right)$. By Theorem 3.8,

$$
\tilde{F} \in \mathfrak{P}_{\frac{p_{n}}{q_{n}} I, n, \varepsilon} \cdot
$$

The result then follows, for $F \in \mathfrak{P}_{I, n, \varepsilon}$ if and only if $\tilde{F} \in \mathfrak{P}_{\frac{p_{n}}{q_{n}} I, n, \varepsilon}$, a consequence of the equality $N^{\triangleleft}(\tilde{F}, \varepsilon) \cap \frac{q_{n}}{p_{n}} I=\frac{q_{n}}{p_{n}}\left(N^{\triangleleft}(F, \varepsilon) \cap I\right)$.

The size of the interval can be shortened in the presence of certain algebraic relations in $F$.

Corollary 3.10. Let $0<\frac{q_{1}}{p}<\cdots<\frac{q_{n}}{p}$ be a finite sequence of fractions in $\mathbb{Q}$ and let $0<\varepsilon<\frac{1}{2}$ be given. Put $N=p q_{n}^{n-2}$.

If $I \subset \mathbb{R}$ is an interval with $\lambda_{\mathbb{R}}(I) \geq N$, then $F:=\left\{\chi_{\frac{q_{j}}{p}}: 1 \leq j \leq n\right\} \in \mathfrak{P}_{I, n, \varepsilon}$.

Proof. We proceed as in Corollary 3.9 and define $\tilde{F}=\frac{p}{q_{n}} F$. Since in this case $\tilde{F}=$ $\left\{\frac{q_{1}}{q_{n}}, \ldots, \frac{q_{n-1}}{q_{n}}, 1\right\}$, we obtain from Theorem 3.8 that $\tilde{F} \in \mathfrak{P}_{I, n, \varepsilon}$, as long as $\lambda_{\mathbb{R}}(I) \geq q_{n}^{n-1}$. The argument of Corollary 3.9 then shows that $F \in \mathfrak{P}_{I, n, \varepsilon}$ if $\lambda_{\mathbb{R}}(I)=p \cdot q_{n}^{n-2}$.

A considerably shorter interval is needed when $F$ is sparse enough:

Theorem 3.11. Fix any $\varepsilon<1 / 2$. Let $F=\left\{\chi_{\tau_{j}}: 1 \leq j \leq n\right\}$ with $2 \varepsilon \tau_{j+1}>\tau_{j}$ for $j=1, \ldots, n-1$.

If $I \subset \mathbb{R}$ is an interval with $\lambda_{\mathbb{R}}(I) \geq \frac{1}{\tau_{1}}$ then $F \in \mathfrak{P}_{I, n, \varepsilon}$.

Proof. Put $I=\left[t_{0}, t_{0}+L_{0}\right]$. Assume first that $L_{0}=\frac{1}{\tau_{1}}$. There is then $I_{1} \subset\left[t_{0}, t_{0}+\frac{1}{\tau_{1}}\right]$ of length $\frac{2 \varepsilon}{\tau_{1}}$ such that $\chi_{\tau_{1}}\left(I_{1}\right)=\mathcal{V}_{\varepsilon}$. Then $\tau_{2}\left(I_{1}\right)=\mathbb{T}$ and we find $I_{2} \subset I_{1}$ of length $2 \varepsilon \cdot \frac{2 \varepsilon}{\tau_{1}}$ with $\chi_{\tau_{2}}\left(I_{2}\right)=\mathcal{V}_{\varepsilon}$. After $n$ steps we find $I_{n} \subseteq I_{n-1} \subset \cdots \subseteq I_{1}$ of length $\frac{(2 \varepsilon)^{n}}{\tau_{1}}$ with $\chi_{\tau_{n}}\left(I_{n}\right) \subset \mathcal{V}_{\varepsilon}$. It follows that $I_{n} \subseteq N^{\triangleleft}(F, \varepsilon) \cap I$ and $F \in \mathfrak{P}_{I, n, \varepsilon}$.

We now proceed as in Theorem 3.9. Find an integer $j>0$ and $0 \leq \delta<\frac{1}{\tau_{1}}$ such that $\lambda_{\mathbb{R}}(I)=\frac{j}{\tau_{1}}+\delta$. Split $I$ in $j$-many subintervals, each of length $\frac{1}{\tau_{1}}$ plus another one of length $\delta$. By the above argument each of the former intervals contains a subinterval $I_{n, l}$, $1 \leq l \leq j$, of length $\frac{(2 \varepsilon)^{n}}{\tau_{1}}$. The proof then goes as in Theorem 3.9. 
Appendix to Section 3. We prove here inequality (3.17) that appears in the proof of Theorem 3.9:

Proof of inequality (3.17).

$$
\varepsilon\left(\left(2\left\lfloor p_{1} \varepsilon\right\rfloor+1\right)\left(2\left\lfloor p_{2} \varepsilon\right\rfloor+1\right) \cdots\left(2\left\lfloor p_{n-1} \varepsilon\right\rfloor+1\right)+1\right) \geq 2 p_{1} \cdots p_{n-1} \varepsilon^{n} .
$$

Since $2\lfloor n \varepsilon\rfloor+1 \geq n \varepsilon$, for every $n \in \mathbb{N}$, it is enough to find $1 \leq k_{1}, k_{2} \leq n-1$ with

$$
\left(2\left\lfloor p_{k_{1}} \varepsilon\right\rfloor+1\right)\left(2\left\lfloor p_{k_{2}} \varepsilon\right\rfloor+1\right) \geq 2 p_{k_{1}} p_{k_{2}} \varepsilon^{2} .
$$

As $2\left\lfloor p_{k_{j}} \varepsilon\right\rfloor+1 \geq 2 p_{k_{j}} \varepsilon-1$, (3.19) will hold as soon as $2 p_{k_{j}} \varepsilon-1 \geq \sqrt{2} p_{k_{j}} \varepsilon$, for some $k_{1} \neq k_{2}$, i.e., as soon as for some $k_{1} \neq k_{2}, p_{k_{j}} \varepsilon \geq \frac{1}{2-\sqrt{2}}$.

On the other hand, if for some $j, 1 \leq p_{k_{j}} \varepsilon \leq \frac{1}{2-\sqrt{2}}$, then $3=\left(2\left\lfloor p_{k_{j}} \varepsilon\right\rfloor+1\right) \geq 2 p_{k_{j}} \varepsilon$ and inequality (3.17) follows.

The only remaining case is when $1>p_{k} \varepsilon$ for every $k$ except at most one $k_{0}$. In that case, using again that, for this special summand, $2\left\lfloor p_{k_{0}} \varepsilon\right\rfloor+1 \geq 2 p_{k_{0}} \varepsilon-1$, we have

$$
\begin{aligned}
\varepsilon\left(\left(2\left\lfloor p_{1} \varepsilon\right\rfloor+1\right)\left(2\left\lfloor p_{2} \varepsilon\right\rfloor+1\right) \cdots\left(2\left\lfloor p_{n-1} \varepsilon\right\rfloor+1\right)+1\right) & =\varepsilon\left(\left(2\left\lfloor p_{k_{0}} \varepsilon\right\rfloor+1\right)+1\right) \\
& \geq \varepsilon\left(p_{1} \varepsilon\right) \cdots\left(2 p_{k_{0}}\right) \cdots\left(p_{n-1} \varepsilon\right) \\
& =2 p_{1} \cdots p_{n-1} \varepsilon^{n} .
\end{aligned}
$$

\section{RANDOM Bohr DEnSE SUbSETS}

The estimates in Section 3 already provide Bohr-density of rather sparse subsets of the real numbers. The argument actually works for the set $\mathfrak{C}_{n}=\left\{\psi_{p}: p \in \mathbb{R}_{n}[x]\right\}$ of generalized chirps of degree at most $n$.

Lemma 4.1. Let $I^{*}=\left\{I_{k}: k \in \mathbb{N}\right\}$ be a sequence of intervals $I_{k}=\left[a_{k}, a_{k}+b_{k}\right]$ in the real numbers with $\lim \sup \left\{b_{k}: k \in \mathbb{N}\right\}=+\infty$. Put $t_{k}:=\max \left(\left|a_{k}\right|,\left|a_{k}+b_{k}\right|\right)$. For each $k$ let $\Delta_{k}$ be a finite subset of $C(G, \mathbb{T})$ whose restrictions to $\left[-t_{k}, t_{k}\right]$ are $\varepsilon_{k}$-dense in $C\left(\left[-t_{k}, t_{k}\right], \mathbb{T}\right)$ with $\lim _{k \rightarrow \infty} \varepsilon_{k}=0$ and consider $\Delta^{*}=\left\{\Delta_{k}: k \in \mathbb{N}\right\}$. Let finally $\ell^{*}=\left\{\ell_{k}: k \in \mathbb{N}\right\}$ be $a$ sequence of positive integers.

If $\left\{d_{s}: s \in \mathbb{N}\right\}$ enumerates a countable dense subset of $\mathbb{R}$ and

$$
\left(\Lambda_{k}\right)_{k} \in \bigcap_{s \in \mathbb{N}} \mathcal{B}_{d_{s}, \Delta^{*}, m, \ell^{*}, \varepsilon, I^{*}}, \quad \text { for some } m \in \mathbb{Z} \text { and } \varepsilon>0
$$

then $\Lambda_{0}:=\bigcup_{k} \Lambda_{k}$ is an $(F, \mathbb{R}, \varepsilon)$-matching set for every subset $F$ of $\mathfrak{C}_{n}$ strongly linearly independent over $\mathbb{Q}$ with $|F|=m$.

Proof. Let $F=\left\{\psi_{p_{1}}, \ldots \psi_{p_{m}}\right\} \subseteq \mathfrak{C}_{n}$ with $\left\{p_{1}(x), \ldots, p_{m}(x)\right\}$ strongly linearly independent over $\mathbb{Q}$.

Fix $x_{0} \in \mathbb{R}$ and consider $N>0$ and $s_{0} \in \mathbb{N}$ with

$$
\begin{gathered}
\frac{3}{N}<\varepsilon, \text { and } \\
d_{a}\left(\psi_{p_{j}}\left(d_{s_{0}}\right), \psi_{p_{j}}\left(x_{0}\right)\right) \leq \frac{1}{N}, \text { for } 1 \leq j \leq n .
\end{gathered}
$$


Since $\left(\Lambda_{k}\right)_{k} \in \mathcal{B}_{d_{s_{0}}, \Delta^{*}, m, \ell^{*}, 1 / N, I^{*}}$, there is $k_{0}$ with

$$
\Lambda_{k} \in \mathcal{A}_{d_{s_{0}}, \Delta_{k}, m, \ell_{k}, 1 / N, I_{k}} \text { for all } k \geq k_{0} .
$$

Apply Theorem 3.6 to $\delta, \gamma>0$ with $\delta<1 / N<2 \delta$ and $(2 \delta)^{m}-\gamma>(1 / N)^{m}$; we can then find $k \geq k_{0}$ large enough to satisfy the following conditions:

$$
\begin{aligned}
b_{k} & >L(F, \gamma) \\
\frac{1}{2}\left(\frac{1}{N}-\delta\right) & \geq \varepsilon_{k} \\
d_{s_{0}} & \in\left[-t_{k}, t_{k}\right] .
\end{aligned}
$$

Note that $b_{k}>L(F, \gamma)$ implies that

$$
\lambda_{\mathbb{R}}\left(N^{\triangleleft}\left(F, \delta, d_{s_{0}}\right) \cap I_{k}\right) \geq\left(\frac{1}{N}\right)^{m} \lambda_{\mathbb{R}}\left(I_{k}\right) .
$$

Since $\Delta_{k}$ is $\varepsilon_{k}$-dense in $C\left(\left[-t_{k}, t_{k}\right], \mathbb{T}\right)$, with the help of (4.24) we can find $\phi_{j} \in \Delta_{k}$, for each $1 \leq j \leq m$, with

$$
d_{a}\left(\phi_{j}(t), \psi_{p_{j}}(t)\right) \leq \frac{1}{2}\left(\frac{1}{N}-\delta\right) \quad \text { for every } t \in\left[-t_{k}, t_{k}\right]
$$

To apply the definition of $\mathcal{A}_{d_{s_{0}}, \Delta_{k}, m, \ell_{k}, 1 / N, I_{k}}$ (Definition 2.7) we need to ensure that $\tilde{F}:=\left\{\phi_{j}: 1 \leq j \leq m\right\}$ is in $\mathfrak{P}_{I_{k}, d_{s_{0}}, m, 1 / N}$. We first show that $N^{\triangleleft}\left(F, \delta, d_{s_{0}}\right) \cap I_{k} \subseteq$ $N^{\triangleleft}\left(\tilde{F}, 1 / N, d_{s_{0}}\right) \cap I_{k}$. Let $t \in N^{\triangleleft}\left(F, \delta, d_{s_{0}}\right) \cap I_{k}$. By (4.21) and (4.27), we have that:

$d_{a}\left(\phi_{j}(t), \phi_{j}\left(d_{s_{0}}\right)\right) \leq d_{a}\left(\phi_{j}(t), \psi_{p_{j}}(t)\right)+d_{a}\left(\psi_{p_{j}}(t), \psi_{p_{j}}\left(d_{s_{0}}\right)\right)+d_{a}\left(\psi_{p_{j}}\left(d_{s_{0}}\right), \phi_{j}\left(d_{s_{0}}\right)\right) \leq \frac{1}{N}$.

Thus $N^{\triangleleft}\left(F, \delta, d_{s_{0}}\right) \cap I_{k} \subseteq N^{\triangleleft}\left(\tilde{F}, 1 / N, d_{s_{0}}\right) \cap I_{k}$. Recalling our choice in (4.26), we conclude that

$$
\tilde{F} \in \mathfrak{P}_{I_{k}, d_{s_{0}}, m, 1 / N}
$$

Applying (4.22) and (4.28) we find $y \in \Lambda_{k}$ with

$$
y \in N^{\triangleleft}\left(\tilde{F}, \frac{1}{N}, d_{s_{0}}\right) .
$$

For this $y$ and each $j, 1 \leq j \leq m$ we have that:

$$
\begin{aligned}
d_{a}\left(\psi_{p_{j}}(y), \psi_{p_{j}}\left(x_{0}\right)\right) & \leq d_{a}\left(\psi_{p_{j}}(y), \psi_{p_{j}}\left(d_{s_{0}}\right)\right)+d_{a}\left(\psi_{p_{j}}\left(d_{s_{0}}\right), \psi_{p_{j}}\left(x_{0}\right)\right) \\
& \leq d_{a}\left(\psi_{p_{j}}(y), \phi_{j}(y)\right)+d_{a}\left(\phi_{j}(y), \phi_{j}\left(d_{s_{0}}\right)\right) \\
& +d_{a}\left(\phi_{j}\left(d_{s_{0}}\right), \psi_{p_{j}}\left(d_{s_{0}}\right)\right)+d_{a}\left(\psi_{p_{j}}\left(d_{s_{0}}\right), \psi_{p_{j}}\left(x_{0}\right)\right) .
\end{aligned}
$$

Now,

by (4.27) and (4.25), and

$$
d_{a}\left(\psi_{p_{j}}(y), \phi_{j}(y)\right)+d_{a}\left(\phi_{j}\left(d_{s_{0}}\right), \psi_{p_{j}}\left(d_{s_{0}}\right)\right)<\frac{1}{N}
$$

$$
d_{a}\left(\psi_{p_{j}}\left(d_{s_{0}}\right), \psi_{p_{j}}\left(x_{0}\right)\right)<\frac{1}{N}
$$

by (4.21). This, together with (4.29), implies that

$$
d_{a}\left(\psi_{p_{j}}(y), \psi_{p_{j}}\left(x_{0}\right)\right) \leq \frac{3}{N}
$$


or, what is the same, that

$$
\psi_{p_{j}}(y) \in \psi_{p_{j}}\left(x_{0}\right) \cdot \mathcal{V}_{\frac{3}{N}} \subset \psi_{p_{j}}\left(x_{0}\right) \cdot \mathcal{V}_{\varepsilon}
$$

which, by (4.20) implies that $\Lambda_{0}$ is an $(F, \mathbb{R}, \varepsilon)$-matching set, as desired.

Theorem 4.2. Let $I^{*}=\left\{I_{k}: k \in \mathbb{N}\right\}$ be a sequence of intervals in the real numbers $I_{k}=\left[a_{k}, a_{k}+b_{k}\right]$ with $\lim \sup \left\{b_{k}: k \in \mathbb{N}\right\}=+\infty$ and such that $t_{k}:=\max \left(\left|a_{k}\right|,\left|a_{k}+b_{k}\right|\right) \geq k$ for every $k$. Pick randomly $\Lambda_{k} \subseteq I_{k}$ with $\left|\Lambda_{k}\right|=\ell_{k}$ and $\ell_{k} \neq O\left(\log \left(t_{k}\right)\right)$. Then, for any fixed $n \in \mathbb{N}, \Lambda:=\bigcup_{k=1}^{\infty} \Lambda_{k}$ is almost surely a set of uniqueness for $\mathcal{A P}_{\mathfrak{C}_{n}}$.

Proof. Fix $n \in \mathbb{N}$. Since $\ell_{k} \neq O\left(\log t_{k}\right)$, the set

$$
\mathcal{K}_{N, m}=\left\{k \in \mathbb{N}: \ell_{k} \geq(n+1) m N^{m} \log 2+\left(m N^{m} \frac{(n+4)(n+1)}{2}-\frac{1}{\log \left(1-\left(\frac{1}{N}\right)^{m}\right)}\right) \log t_{k}\right\}
$$

is infinite for each $N, m \in \mathbb{N}$.

Define for each $k \in \mathbb{N}$,

$$
\widetilde{\Delta_{k}}=\left\{\sum_{i=0}^{n} \frac{j_{i}}{t_{k}^{i+1}} x^{i}:-\left\lfloor t_{k}^{i+2}\right\rfloor \leq j_{i}<\left\lfloor t_{k}^{i+2}\right\rfloor, \quad j_{i} \in \mathbb{Z}, 0 \leq i \leq n\right\} \subseteq \mathfrak{C}_{n}
$$

and

$$
\Delta_{k}=\left\{\psi_{p}: p(t) \in \widetilde{\Delta_{k}}\right\}
$$

Observe that $\left|\Delta_{k}\right|=\prod_{i=0}^{n}\left(2\left\lfloor t_{k}^{(i+2)}\right\rfloor\right) \leq 2^{n+1} t_{k}^{\frac{(n+1)(n+4)}{2}}$.

Fix $m, N \in \mathbb{N}$. If $k \in \mathcal{K}_{N, m}$,

$$
\begin{aligned}
\ell_{k} & \geq(n+1) m N^{m} \log 2+\left(m N^{m} \frac{(n+4)(n+1)}{2}-\frac{1}{\log \left(1-\left(\frac{1}{N}\right)^{m}\right)}\right) \log t_{k} \\
& \geq m N^{m} \log \left|\Delta_{k}\right|-\left(\frac{1}{\log \left(1-\left(\frac{1}{N}\right)^{m}\right)}\right) \log k .
\end{aligned}
$$

Lemma 2.12 applied to $I_{N, m}^{*}=\left\{I_{k}: k \in \mathcal{K}_{N, m}\right\}, \Delta_{N, m}^{*}=\left\{\Delta_{k}: k \in \mathcal{K}_{N, m}\right\}$ and $\ell_{N, m}^{*}=$ $\left\{\ell_{k}: k \in \mathcal{K}_{N, m}\right\}$ yields then that

$$
\mathbb{P}\left(\mathcal{B}_{q, \Delta_{N, m}^{*}, m, \ell_{N, m}^{*}, 1 / N, I_{N, m}^{*}}\right)=1
$$

for every $q \in \mathbb{R}$.

Let $D=\left\{d_{s}: s \in \mathbb{N}\right\}$ be a countable dense subset of $\mathbb{R}$. The event

$$
\mathcal{B}_{0}=\bigcap_{s \in \mathbb{N}} \bigcap_{m \in \mathbb{N}} \bigcap_{N \in \mathbb{N}} \mathcal{B}_{d_{s}, \Delta_{N, m}^{*}, m, \ell_{N, m}^{*}, 1 / N, I_{N, m}^{*}}
$$

then occurs with probability one.

Let $\left(\Lambda_{k}\right)_{k} \in \mathcal{B}_{0}$ and let $\Lambda_{0}=\bigcup_{k} \Lambda_{k}$. We now prove that $\Lambda_{0}$ is an $(F, \mathbb{R}, \varepsilon)$-matching for every $n$, every finite set $F \subset \mathfrak{C}_{n}$ and every $\varepsilon>0$.

Fix therefore $\varepsilon>0$ and a finite set of polynomials $\left\{p_{1}(x), \ldots, p_{s}(x)\right\} \subset \mathbb{R}_{n}[x]$. Let $F=\left\{\psi_{p_{1}}, \ldots, \psi_{p_{s}}\right\}$. We may assume that $\left\{p_{1}, \ldots, p_{m}\right\}$ are strongly linearly independent 
over $\mathbb{Q}$ and that, for each $j=m+1, \ldots, s$, there are a constant $C_{j} \in \mathbb{R}$ and integers $Q$ and $Z_{i j}, 1 \leq i \leq m$ with

$$
p_{j}=C_{j}-\sum_{i=1}^{m} \frac{Z_{i j}}{Q} p_{i} .
$$

Then $\tilde{F}=\left\{\frac{p_{1}}{Q}, \ldots, \frac{p_{m}}{Q}\right\}$ is strongly linearly independent over $\mathbb{Q}$. Define

$$
M=\max \left\{\sum_{i=1}^{m}\left|Z_{i j}\right|: m+1 \leq j \leq n\right\} .
$$

and $\tilde{\varepsilon}=\frac{\varepsilon}{M}$. Fix $N$ with $1 / N<\tilde{\varepsilon}$.

We next apply Lemma 4.1 to the sequences $I_{N, m}^{*}, \Delta_{N, m}^{*}$ and $\ell_{N, m}^{*}$. Observe that this can be done as $\Delta_{k}$ is $\frac{n+1}{t_{k}}$-dense in the restrictions of $\mathfrak{C}_{n}$ to $\left[-t_{k}, t_{k}\right]$. Since $\left(\Lambda_{k}\right)_{k} \in \bigcap_{s \in \mathbb{N}} \mathcal{B}_{d_{s}, \Delta_{N, m}^{*}, m, \ell_{N, m}^{*}, 1 / N, I_{N, m}^{*}}$, we deduce that $\Lambda_{0}$ is an $(\tilde{F}, \mathbb{R}, \tilde{\varepsilon})$-matching set.

We finally check that $\Lambda_{0}$ is an $(F, \mathbb{R}, \varepsilon)$-matching set.

Let $a \in \mathbb{R}$. Since $\Lambda_{0}$ is a $(\tilde{F}, \mathbb{R}, \tilde{\varepsilon})$-matching set, there is $x_{a} \in \Lambda_{0}$ such that $x_{a} \in$ $N^{\triangleleft}(\tilde{F}, \tilde{\varepsilon}, a)$, that is, for each $i=1, \ldots, m$ there are $\delta_{i}$ with $\left|\delta_{i}\right|<\tilde{\varepsilon}$ and $M_{i} \in \mathbb{Z}$ with

$$
\frac{p_{i}}{Q}\left(x_{a}\right)-\frac{p_{i}}{Q}(a)=\delta_{i}+M_{i}
$$

Then, for $j=m+1, \ldots, s$,

$$
\begin{aligned}
p_{j}\left(x_{a}\right)-p_{j}(a) & =\sum_{i=1}^{m} Z_{i j}\left(\frac{p_{i}}{Q}\left(x_{a}\right)-\frac{p_{i}}{Q}(a)\right) \\
& =\sum_{i=1}^{m} Z_{i j}\left(\delta_{i}+M_{i}\right) .
\end{aligned}
$$

Since $\sum_{i=1}^{m} Z_{i j} M_{i} \in \mathbb{Z}$ and $\left|\sum_{i=1}^{m} Z_{i j} \delta_{i}\right| \leq \varepsilon$ we see that, for $j=m+1, \ldots, s$,

$$
\psi_{p_{j}}\left(x_{a}\right) \in \psi_{p_{j}}(a) \cdot \mathcal{V}_{\varepsilon}
$$

The same conclusion being obvious for $j=1, \ldots, m$, it follows that $x_{a} \in N^{\triangleleft}(F, \varepsilon, a)$, as we wanted to show.

$\Lambda_{0}$ is thus an $(F, \mathbb{R}, \varepsilon)$-matching set for every $\varepsilon>0$ and every finite set $F \subset \mathfrak{C}_{n}$. Corollary 2.5 then implies the property we had to prove.

In the case of $\mathcal{A P}(\mathbb{R})$, we also obtain almost sure density.

Theorem 4.3. Let $I^{*}=\left\{I_{k}: k \in \mathbb{N}\right\}$ be a sequence of intervals in the real numbers $I_{k}=\left[a_{k}, a_{k}+b_{k}\right]$ with $\lim \sup \left\{b_{k}: k \in \mathbb{N}\right\}=+\infty$ and such that $t_{k}:=\max \left(\left|a_{k}\right|,\left|a_{k}+b_{k}\right|\right) \geq k$ for every $k$. Pick randomly $\Lambda_{k} \subseteq I_{k}$ with $\left|\Lambda_{k}\right|=\ell_{k}$ and $\ell_{k} \neq O\left(\log \left(t_{k}\right)\right)$. Then, almost surely, $\Lambda:=\bigcup_{k=1}^{\infty} \Lambda_{k}$ is dense in $\mathbb{R}^{\mathcal{A P}}$.

The same argument yields the following general version of Theorem 3.1 in [9].

Theorem 4.4. Let $I^{*}=\left\{I_{k}: k \in \mathbb{N}\right\}$ be a sequence of intervals in the integers, $I_{k}=$ $\left[n_{k}, n_{k}+m_{k}\right]$ with $\lim \sup \left\{m_{k}: k \in \mathbb{N}\right\}=+\infty$ and such that $t_{k}:=\max \left(\left|n_{k}\right|,\left|n_{k}+m_{k}\right|\right) \geq k$ for every $k$. Pick randomly $\Lambda_{k} \subseteq I_{k}$ with $\left|\Lambda_{k}\right|=\ell_{k}$ and $\ell_{k} \neq O\left(\log \left(t_{k}\right)\right)$. Then, almost surely $\Lambda:=\bigcup_{k=1}^{\infty} \Lambda_{k}$ is dense in $\mathbb{Z}^{\mathcal{A P}}$. 


\section{SAMPling IN A Finite INTERVAL}

As we have explored in the previous sections, taking a few points in a series of increasingly long intervals almost surely produces a Bohr-dense subset. This yields very sparse sets of uniqueness for almost periodic functions.

In the present section, we make a first attempt at quantifying these results. We estimate how large an interval must be and how many sampling points must be taken to be sure that with high probability the resulting finite set determines (approximately) a simple family of almost periodic functions (namely, trigonometric polynomials of bounded degree).

Setting. We shall consider an interval $J \subseteq \mathbb{R}, J=[t, t+L], t, L \geq 0$, a discretized band of frequencies $\Delta=\left\{\chi_{\frac{1}{M}}, \chi_{\frac{2}{M}}, \ldots, \chi_{\frac{\Omega M}{M}}=\chi_{\Omega}\right\} \subset \widehat{\mathbb{R}}$ with $\Omega \geq 1$ and some $n \in \mathbb{N}$. We assume for simplicity that $L, \Omega \in \mathbb{N}$. We then take a random subset $\Lambda \subseteq J$ with $|\Lambda|=\ell$. We say that our choice has succeeded if $\Lambda$ is a $(F, J, \varepsilon)$-matching set for every finite subset of $\Delta$ with $|F|=n$. Our aim is to determine $L$ and $\ell$ so that the probability of success is as large as $1-\delta$ (with $\delta$ close to zero). Via Proposition 2.4 , this will produce, with probability $1-\delta$, a $(6 \pi+2 \varepsilon)$-sampling set for those (periodic-type) functions having precisely $n$ frequency components that belong to $\Delta$.

Proposition 5.1. Let $J$ be an interval in $\mathbb{R}, \Delta \subseteq \widehat{\mathbb{R}}$ a set of frequencies, $\Lambda \subseteq J$ and $n$ be as above. Let $N>4$. If $L \geq M \Omega^{n-2}$, then $\Lambda$ will be an $(F, J, 2 / N)$-matching set for every $F \subset \Delta$ such that $|F|=n$, with probability at least

$$
1-N(t+L)\left(\begin{array}{c}
\Omega M \\
n
\end{array}\right)\left(1-\left(\frac{1}{N}\right)^{n}\right)^{\ell}
$$

Proof. Let $K=N(t+L)$ and take $K$ equally spaced points $\left\{t_{0}, t_{1}, \ldots, t_{K-1}\right\}$ in $J$. We first check that if $\Lambda \in \bigcap_{j=0}^{K-1} \mathcal{A}_{t_{j}, \Delta, n, \ell, 1 / N, J}$, then $\Lambda$ is an $(F, J, 2 / N)$-matching set for every $F \subset \Delta$ with $|F|=n$.

Consider $F \subseteq \Delta$ with $|F|=n$ and let $a \in J$. We find $j$ with $\left|t_{j}-a\right|<1 / K$. By Corollary 3.10, $F \in \mathfrak{P}_{I, n, 1 / N}$. Since $\Lambda \in \mathcal{A}_{t_{j}, \Delta, n, \ell, 1 / N, I}$, we see that there is $x \in \Lambda$ such that $x \in N^{\triangleleft}\left(F, 1 / N, t_{j}\right)$. It is then clear that $x \in N^{\triangleleft}(F, 2 / N, a)$. $\Lambda$ is thus a $(F, J, 2 / N)$ matching set.

Now, according to Lemma 2.9,

$$
\mathbb{P}\left(\bigcap_{j=0}^{K-1} \mathcal{A}_{t_{j}, \Delta, n, \ell, 2 / N, I}\right) \geq 1-K\left(\begin{array}{c}
\Omega M \\
n
\end{array}\right)\left(1-\left(\frac{1}{N}\right)^{n}\right)^{\ell} .
$$

Proposition 5.2. Let $J \subseteq \mathbb{R}, \Delta \subseteq \widehat{\mathbb{R}}$ and $n$ be as established in the setting of this section. Suppose $\Lambda \subseteq J$ with $|\Lambda|=\ell$ is randomly extracted. The probability of $\Lambda$ to be an $\left(F, J, \frac{2}{N}\right)$-matching set will be at least $1-\delta$ provided the following conditions hold:

(1) The length of $J$ is at least $M \Omega^{n-2}$.

(2) $\ell \geq N^{n}(\log N+\log (t+L)+n \log \Omega+n \log M-\log \delta)$.

Proof. Taking logarithms in the estimate of Proposition 5.1 we see that the required probability $(1-\delta)$ will be attained provided

$$
\log \left(N(t+L)\left(\begin{array}{c}
\Omega M \\
n
\end{array}\right)\left(1-\left(\frac{1}{N}\right)^{n}\right)^{\ell}\right) \leq \log \delta
$$


Recalling that $\log \left(1-\left(\frac{1}{N}\right)^{n}\right)<-\left(\frac{1}{N}\right)^{n}$ and that $\left(\begin{array}{l}h \\ k\end{array}\right) \leq\left(\frac{h e}{k}\right)^{k}$ for every pair of integers $h$ and $k$, it follows that to reach probability $(1-\delta)$ it is enough that

$$
\ell \geq N^{n}\left(\log (N(t+L))+n \log \left(\frac{\Omega M e}{n}\right)-\log \delta\right)
$$

thence statement (2).

The above proposition shows that our estimates ensure good sampling properties as long as the sampling interval is long enough. In that case the sampling ratio can be made as small as desired, for the number of samples depends chiefly on the accuracy required in the approximate sampling. The rest of factors (number and width of frequencies, the desired reliability in terms of the probability of success) have, in comparison, a low impact.

Example 5.3. Suppose that we work in the interval $J=[0, L]$, that $\Delta$ is determined by $M=100, \Omega=1$ and that $n=3$, i.e., that our functions are linear combinations of at most three characters $\chi_{\tau}$ with $\tau=\frac{j}{100}, 0 \leq j \leq 99$. For the probability of success to be at least $1-\delta$ and the precision to be $\varepsilon=1 / N$, it will be enough that $L \geq 100$ and that the number of sample points, say $\ell$, is at least:

$$
\ell \geq N^{3}(14+\log N+\log L-\log \delta) .
$$

So, once the desired precision $1 / N$ and reliability $1-\delta$ are fixed and as long as the length of the sampling interval is at least 100, the number of samples increases as $\mathrm{O}\left(N^{3} \log N\right)$.

If we fix $N$ and let $M$ get larger (keeping $\Omega=1, n=3$ ) we have that

$$
\ell \geq N^{3}(\log L+\log N+3 \log M-\log \delta)
$$

and, eventually, $\ell$ gets small with respect to both $M$ and the sampling length.

\section{Obtaining Bohr-Dense sets With Special Properties}

The estimates of Section 2 can be easily used to find Bohr-dense sets with special properties, as long as the conditions imposed in Lemma 2.12 and Theorem 4.2 leave enough room for a random subset to satisfy the required properties.

In this section we focus on interpolation properties of sets. For a given algebra $\mathcal{A} \subset$ $C(G, \mathbb{C})$, with $G$ a topological group, a subset $X \subseteq G$ is said to be an $\mathcal{A}$-interpolation set if every bounded function $f: X \rightarrow \mathbb{C}$ admits a continuous extension $\tilde{f}: G \rightarrow \mathbb{C}$ with $\tilde{f} \in \mathcal{A}$. We introduce now a class of sets that, in addition to the combinatorial flavour of their definition, turn out to be sets of interpolation for the algebra $\mathrm{WAP}(G)$ of weakly almost periodic functions.

Definition 6.1. Let $G$ be a topological group. A subset $E$ of $G$ is a $t$-set if for every $g \in G, g \neq 0$, the intersection $E \cap(E+g)$ is relatively compact.

Rudin [14] introduced $t$-sets and proved that every function supported on a $\mathrm{t}$-set of a discrete group is automatically weakly almost periodic, see [5] for further references on this topic including the definition of weakly almost periodic function. In the terminology of [5], t-sets in LCA groups are (approximable) WAP $(G)$-interpolation sets.

Sets of interpolation for the Fourier-Stieltjes algebra $B(G)$ (consisting of FourierStieltjes transforms of measures on $\widehat{G}$ ) are known as Sidon sets and have been heavily studied (see the monographs [12] and [6]). Since $B(G) \subseteq \mathrm{WAP}(G)$, we have that both Sidon sets and $t$-sets are sets of interpolation for the algebra of weakly almost periodic 
functions. An interesting observation is that every Sidon set can be decomposed as a finite union of t-sets (see [6, Corollary 6.4.7]).

As already noted in [9], the random process of Theorems 4.2 and 4.4 cannot be adapted to work with Sidon sets. It is well known, see e.g. [6, Corollary 6.3.13] that an interval $[a, a+N]$ cannot contain more than $C_{E} \log N$ elements of a Sidon set $E$. This route has however proved to be fruitful with other less demanding properties: Neuwirth [13], for instance, obtains dense subsets of $\mathbb{Z}^{\mathcal{A P}}$ that are $\Lambda(p)$ for every $p$, and Li, Piazza and Queffelec [11] have obtained dense subsets of $\mathbb{Z}^{\mathcal{A P}}$ that are $p$-Sidon for every $p>1$. We do not need these concepts here and we refer to these papers, or to [6], for their proper definitions. It suffices to say that Sidon sets are $p$-Sidon for every $p>1$ and $\Lambda(p)$-sets for every $p$.

While, as mentioned, our construction does not work with Sidon sets, it does work with the important class of $t$-sets. We show in this section that t-sets that are dense in $G^{\mathcal{A P}}$ do exist for $G=\mathbb{Z}$ and $G=\mathbb{R}$ and that, indeed, random constructions in the spirit of Lemma 2.12 lead almost surely to t-sets that are dense in $G^{\mathcal{A P}}$.

We first need a lemma that helps in recognizing t-sets. For a subset $\Lambda \subset \mathbb{R}$, we define its step length as

$$
\operatorname{StL}(\Lambda)=\inf \left\{\left|z-z^{\prime}\right|: z, z^{\prime} \in \Lambda, z \neq z^{\prime}\right\} .
$$

Lemma 6.2. For each $k \in \mathbb{N}$, let $\Lambda_{k}$ be a finite subset of the interval $\left[a_{k}, b_{k}\right] \subset \mathbb{R}$. Suppose that the collection of sets $\Lambda_{k}, k \in \mathbb{N}$, and the corresponding sequence of intervals $\left[a_{k}, b_{k}\right]$ are constructed in such a way that the following conditions hold:

(1) The sequence of gaps $\left(a_{k+1}-b_{k}\right)_{k}$ is increasing and unbounded.

(2) There is $k_{0}$ such that $\operatorname{StL}\left(\Lambda_{k}\right)>b_{k-1}-a_{k-1}$ for any $k \geq k_{0}$.

Then $\Lambda=\bigcup_{k} \Lambda_{k}$ is a $t$-set.

Proof. Suppose there is $0 \neq t_{0} \in \mathbb{R}$ such that $\Lambda \cap\left(\Lambda+t_{0}\right)$ is unbounded.

Since the sets $\Lambda_{k}$ are finite, there will be $k<k^{\prime}$, with $k>k_{0}, a_{k}-b_{k-1}>\left|t_{0}\right|$ and $t_{k} \in \Lambda_{k} \cap\left(\Lambda+t_{0}\right), t_{k^{\prime}} \in \Lambda_{k^{\prime}} \cap\left(\Lambda+t_{0}\right)$. Then $t_{0}=t_{k}-t_{1}=t_{k^{\prime}}-t_{2}$ with $t_{1}, t_{2} \in \Lambda$. If $t_{0}>0$, using that $t_{0}<a_{k}-b_{k-1}<a_{k^{\prime}}-b_{k^{\prime}-1}$, one has that $b_{k-1}<t_{1} \leq t_{k}$ and that $b_{k^{\prime}-1}<t_{2} \leq t_{k^{\prime}}$. A symmetric argument works when $t_{0}<0$. It follows that $t_{1} \in \Lambda_{k}$ and $t_{2} \in \Lambda_{k^{\prime}}$. But then $\operatorname{StL}\left(\Lambda_{k^{\prime}}\right) \leq\left|t_{k^{\prime}}-t_{2}\right|=\left|t_{0}\right|<\left|a_{k}-b_{k-1}\right|$, a contradiction with hypothesis (2).

Theorem 6.3. Let $I^{*}=\left\{I_{k}: k \in \mathbb{N}\right\}$ be a sequence of intervals in $\mathbb{Z}, I_{k}=\left[z_{k}, z_{k}+N_{k}\right]$ such that $\left(z_{k+1}-z_{k}-N_{k}\right)_{k}$ is increasing and unbounded. Pick randomly $\Lambda_{k} \subseteq I_{k}$ with $\left|\Lambda_{k}\right|=\ell_{k}$. If $\sum_{k=1}^{\infty} \frac{\ell_{k}^{2} N_{k-1}}{N_{k}}<\infty$, then, almost surely, $\Lambda:=\bigcup_{k=1}^{\infty} \Lambda_{k}$ is a $t$-set.

Proof. In the usual probability space, fixed in Subsection 1.1.6, consider for each $k$ the event:

$$
\mathcal{B}_{k}=\left\{\Lambda_{k}: \operatorname{StL}\left(\Lambda_{k}\right) \leq N_{k-1}\right\} .
$$

A rough estimate of the probability of this event is obtained by observing that any choice of $\ell_{k}$ elements with step length less than $N_{k-1}$ is witnessed by two elements $I_{k}$ at a distance of at most $N_{k-1}$. If for each element $z$ in $I_{k}$ we find those elements in $I_{k}$ larger than $z$ but within a distance of at most $N_{k-1}$, we see that there are at most $N_{k-1}$ possible witnesses containing $z$. Since there are at most $\left(\begin{array}{c}N_{k}-2 \\ \ell_{k}-2\end{array}\right)$ different subsets of $I_{k}$ of cardinality $\ell_{k}$ that 
contain a given witness, we deduce altogether that:

$$
\mathbb{P}\left(\mathcal{B}_{k}\right) \leq \frac{\left(N_{k}-1\right) N_{k-1}\left(\begin{array}{c}
N_{k}-2 \\
\ell_{k}-2
\end{array}\right)}{\left(\begin{array}{l}
N_{k} \\
\ell_{k}
\end{array}\right)}=\frac{\ell_{k}\left(\ell_{k}-1\right) N_{k-1}}{N_{k}}
$$

We deduce from our hypothesis on the sequence $\left(\frac{\ell_{k}^{2} N_{k-1}}{N_{k}}\right)_{k}$ that $\sum_{k} \mathbb{P}\left(\mathcal{B}_{k}\right)<\infty$. The Borel-Cantelli Lemma then shows that almost surely there is $k_{0} \in \mathbb{N}$ such that for every $k \geq k_{0}, \Lambda_{k} \notin \mathcal{B}_{k}$. Lemma 6.2 then proves that $\Lambda$ is a t-set.

Theorem 6.4. Let $I^{*}=\left\{I_{k}: k \in \mathbb{N}\right\}$ be a sequence of intervals in $\mathbb{R}, I_{k}=\left[z_{k}, z_{k}+N_{k}\right]$ such that $\left(z_{k+1}-z_{k}-N_{k}\right)_{k}$ is increasing and unbounded. Pick randomly $\Lambda_{k} \subseteq I_{k}$ with $\left|\Lambda_{k}\right|=\ell_{k}$. If $\sum_{k=1}^{\infty} \frac{\ell_{k}^{2} N_{k-1}}{N_{k}}<\infty$, then, almost surely, $\Lambda:=\bigcup_{k=1}^{\infty} \Lambda_{k}$ is a t-set.

Proof. Again in the probabilistic framework of Subsection 1.1.6, consider, for each $k$, the event:

$$
\mathcal{B}_{k}=\left\{\Lambda_{k}: \operatorname{StL}\left(\Lambda_{k}\right) \leq N_{k-1}\right\} .
$$

We first estimate the probability of $\mathcal{B}_{k}$ when $\ell_{k}=2$. In this case,

$$
\mathcal{B}_{0}=\left\{\{x, y\} \subset I_{k}:|x-y| \leq N_{k-1}\right\} .
$$

Once $x \in I_{k}$ is chosen, the probability of $\{x, y\}$ to be in $\mathcal{B}_{0}$ follows the following function:

$$
\left.h(x)=\mathbb{P}\left(\left\{y \in I_{k}:\{x, y\}\right) \in \mathcal{B}_{0}\right\}\right)= \begin{cases}\frac{N_{k-1}+\left(x-z_{k}\right)}{N_{k}} & \text { if } x<z_{k}+N_{k-1}, \\ \frac{2 N_{k-1}}{N_{k}} & \text { if } z_{k}+N_{k-1} \leq x \leq z_{k}+N_{k}-N_{k-1}, \\ \frac{N_{k-1}+\left(z_{k}+N_{k}-x\right)}{N_{k}} & \text { if } z_{k}+N_{k}-N_{k-1}<x \leq z_{k}+N_{k} .\end{cases}
$$

As a consequence,

$$
\mathbb{P}\left(\mathcal{B}_{0}\right)=\frac{N_{k-1}}{N_{k}^{2}}\left(2 N_{k}-N_{k-1}\right) .
$$

Now, if a set $\Lambda_{k}$ consisting of $\ell_{k}$ points is chosen in $I_{k}$, for $\Lambda_{k}$ to be in $\mathcal{B}_{k}$ it will be enough that any pair of its elements, say $\{x, y\}$ satisfies $|x-y| \leq N_{k-1}$. A rough estimate is then:

$$
\mathbb{P}\left(\mathcal{B}_{k}\right) \leq\left(\begin{array}{c}
\ell_{k} \\
2
\end{array}\right) \cdot \mathbb{P}\left(\mathcal{B}_{0}\right)=\frac{\ell_{k}\left(\ell_{k}-1\right) N_{k-1}}{2 N_{k}^{2}}\left(2 N_{k}-N_{k-1}\right) \leq \frac{\ell_{k}^{2} N_{k-1}}{N_{k}} .
$$

We deduce from our hypothesis on $\left(\frac{\ell_{k}^{2} N_{k-1}}{N_{k}}\right)_{k}$ that $\sum \mathbb{P}\left(\mathcal{B}_{k}\right)<\infty$. The Borel-Cantelli Lemma then shows that almost surely there is $k_{0} \in \mathbb{N}$ such that for every $k \geq k_{0}, \Lambda_{k} \notin \mathcal{B}_{k}$.

Lemma 6.2 proves then that $\Lambda$ is a t-set.

We now choose parameters in Theorem 4.2 so as to fit in Theorem 6.3.

Theorem 6.5. Let $I^{*}=\left\{I_{k}: k \in \mathbb{N}\right\}$ be a sequence of intervals in $\mathbb{R}, I_{k}=\left[L_{k}, 2 L_{k}\right]$ with $L_{k}=(k !)^{4}$ for every $k$. Pick randomly $\Lambda_{k} \subseteq I_{k}$ with $\left|\Lambda_{k}\right|=k$. Then, almost surely, $\Lambda:=\bigcup_{k=1}^{\infty} \Lambda_{k}$ is a dense $t$-set in $\mathbb{R}^{\mathcal{A P}}$. If we choose $\Lambda_{k} \subset \mathbb{Z}$, then, almost surely, $\Lambda$ is a dense $t$-set in $\mathbb{Z}^{\mathcal{A P}}$.

Proof. This sequence of intervals satisfies the hypothesis of both Theorems 4.2 and 6.4, or their analogs 4.4 and 6.3. Hence $\Lambda$ almost surely satisfies both conclusions. 
The sets of Theorems 4.4 and 6.5 have asymptotic density zero. Other examples of Bohr-dense subsets of $\mathbb{Z}$ are obtained in [1]. The sets in [1] satisfy the condition:

$$
\lim _{N \rightarrow \infty} \frac{1}{N}\left|E_{N} \cap\left(E_{N}+k\right)\right|=1, \text { for all } k \in \mathbb{Z},
$$

where $E_{N}$ is the set consisting of the first $N$ terms of $E$. They are therefore very far from being t-sets.

\section{REFERENCES}

[1] J. R. Blum, B. Eisenberg, and L.-S. Hahn, Ergodic theory and the measure of sets in the Bohr group, Acta Sci. Math. (Szeged) 34 (1973), 17-24.

[2] E. Carlen and R. V. Mendes, Signal reconstruction by random sampling in chirp space, Nonlinear Dynam. 56 (2009), no. 3, 223-229.

[3] P. Collet, Sampling almost-periodic functions with random probes of finite density, Proc. Roy. Soc. London Ser. A 452 (1996), no. 1953, 2263-2277.

[4] M. Drmota and R. F. Tichy, Sequences, discrepancies and applications, Lecture Notes in Mathematics, vol. 1651, Springer-Verlag, Berlin, 1997.

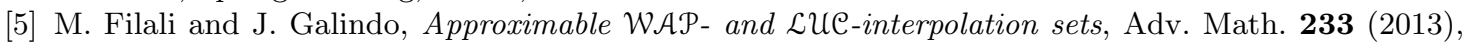
$87-114$.

[6] C. C. Graham and K. E. Hare, Interpolation and Sidon sets for compact groups, CMS Books in Mathematics/Ouvrages de Mathématiques de la SMC, Springer, New York, 2013.

[7] J. P. Kahane and Yitzhak Katznelson, Distribution uniforme de certaines suites d'entiers aléatoires dans le groupe de Bohr, J. Anal. Math. 105 (2008), 379-382.

[8] Y. Katznelson, An introduction to harmonic analysis, corrected ed., Dover Publications Inc., New York, 1976.

[9] Y. Katznelson, Sequences of integers dense in the Bohr group, Proc. Roy. Inst. of Tech. (June 1973), 79-86.

[10] L. Kuipers and H. Niederreiter, Uniform distribution of sequences, Wiley-Interscience [John Wiley \& Sons], New York-London-Sydney, 1974, Pure and Applied Mathematics.

[11] D. Li, H. Queffélec, and L. Rodríguez-Piazza, Some new thin sets of integers in harmonic analysis, J. Anal. Math. 86 (2002), 105-138.

[12] J. M. López and K. A. Ross, Sidon sets, Marcel Dekker Inc., New York, 1975, Lecture Notes in Pure and Applied Mathematics, Vol. 13.

[13] S. Neuwirth, Two random constructions inside lacunary sets, Ann. Inst. Fourier (Grenoble) 49 (1999), no. $6,1853-1867$.

[14] W. Rudin, Weak almost periodic functions and Fourier-Stieltjes transforms, Duke Math. J. 26 (1959), $215-220$.

[15] _ Fourier analysis on groups, John Wiley \& Sons Inc., New York, 1990, Reprint of the 1962 original, A Wiley-Interscience Publication.

[16] I. E. Segal, The class of functions which are absolutely convergent Fourier transforms, Acta Sci. Math. Szeged 12 (1950), no. Leopoldo Fejér et Frederico Riesz LXX annos natis dedicatus, Pars B, 157-161.

Stefano Ferri, Departamento de Matemáticas, Universidad de los Andes, Carrera 1.a 18 A 10, Bogotá D.C., Colombia. Apartado Aéreo 4976.

E-MAIL: stferri@uniandes.edu.co

Jorge Galindo, Instituto Universitario de Matemáticas y Aplicaciones (IMAC), UniversiDAD Jaume I, E-12071, CASTellón, Spain.

E-MAIL: jgalindo@uji.es

Camilo Gómez, Departamento de Matemáticas, Universidad de los Andes, Carrera 1.a 18 A 10, Bogotá D.C., Colombia. Apartado Aéreo 4976. Facultad de Ingeniería, Universidad de la Sabana, Campus Universitario Puente del Común, Chía, Colombia. Departamento de Matemáticas, Escuela Colombiana de Ingeniería, AK 45 205-59, Bogotá D.C., Colombia.

E-MAIL: alfon-go@uniandes.edu.co 